\title{
Les appareils générateurs de houle en laboratoire
}

\section{Laboratory wave generating apparatus}

\section{ETUDE THÉORIQUE D'UN CERTAIN TYPE D'APPAREIL A HOULE THEORETICAL STUDY OF A CERTAIN TYPE OF WAVE MACHINE}

\author{
PAR F. BIESEL ET F. SUQUET \\ ET UN GROUPE D'INGENIEURS AU LABORATOIRE DAUPHINOIS D'HYDRAUROUE (NEYRPIC, GRENOBLE)
} (Suite)

(CE. Ia Honille Blanche, n" 2, 1951, page 147.)

English synopsis, p. 462.

\section{Rappel du Sommaire}

\section{I. - INTRODUCTION}

$1^{\circ}$ Définition du problème étudié et notations.

$2^{\circ}$ Théories antérieures.

$$
\text { II. - DÉTAII DU CAICCUL. }
$$

1. Etude du problème homogène.

$2^{\circ}$ Recherche d'une solution particulière.

$3^{\circ}$ Détermination de la solulion définilive.

\section{III. - DISCUSSION DES RÉSULTATS}

10 Indications générales.

$2^{\circ}$ Etude de quelques cas particulier's.

A. - Batteux piston :

a) Batteur piston émettant des houles longues.

b) Batteur piston émettant des houles courtes.

B. .... Volet battant :

a) Volet battant émetiant des houles longues.

b) Volet battant émettant des houles courtes.

\section{IV. - CONCLUSIONS GÉNÉRALES}

(Les $3^{\circ}$ et $4^{\circ}$ parties sont publiees dans le présent numéro.)
1" Fxistence de la solution du probleme de décomposition posé par l'équation 16.

$2^{\circ}$ Etude particulière des coefficients $c_{n}$ :

$3^{\circ}$ Justification des démonstrations formelles données dans le texle de la note.

4. Elude des points singuliers.

$5^{\circ}$ Justification des condilions aux limiles.
(Les deux premieres parties ont été publiées dans le $n^{0}$ ? 1951.) 


\section{III. - Discussion des résultats}

La discussion qualitative de la formule (22) est vite faite. On voit que le mouvement imprimé par le batteur à l'eau se compose d'une houle ordinaire, telle que l'émettrait un batteur idéal, et d'une perturbation initiale, ou transitoire dans l'espace, qui décroît à peu près exponentiellement lorsque l'on s'éloigne du batteur.

La discussion quantitalive est plus complexe car les résultats varient suivant la forme du batteur. On ne peut, par exemple, tirer de conclusion générale en ce qui concerne le rapport entre lamplitude de la houle et celle des perturbations puisque, pour certaines formes de la fonction $\xi(y)$, cette houle peut cesser completement d'exister. Nous nous contenterons done, dans ce qui suit, de donner quelques indications sommaires sur le cas général et nous terminerons en développant complètement quelques exemples particuliers.

\section{Indications gént́rales.}

La perturbation initiale se compose d'une somme de termes dont chacun décroît exponentiellement comme $e^{-m_{n} x}$. Le terme décroissant le plus lentement sera le premier $e^{-m_{1} x}$.

On peut déterminer graphiquement les valeurs des coefficients $m_{n}$, ou plutòt celle des produits sans dimensions $m_{n} h$. Ces dernières valeurs seront les abseisses des intersections des courbes d'équations :

$\beta=\frac{g}{k^{2} h} \quad \alpha=\frac{\alpha}{m h \operatorname{th} m h}$ et $\beta=-\operatorname{ctg} \alpha$

Ces courbes sont tracées sur la figure 4 qui permet de voir aisément que l'on a toujours $m_{1}>\frac{\pi}{2 h}$, par conséquent les perturbations décroîtront au moins aussi vite que $e^{-\frac{\pi}{2 h t} x}$. A une distance du batteur égale à la profondeux, elles n'auront plus au maximum que $21 \%$ de leur amplitude initiale, à une distance égale à deux fois la profondeur plus que $4,3 \%$ et a une distance égale à trois fois la profondeur plus que $1 \%$.

On voit aisément que le terme en $e^{-m_{2} x}$ décroit déjà beaucoup plus rapidement puisque l'on a : $m_{2}>\frac{3 \pi}{2 h}$. Cette deuxième composante est réduite dans le rapport de 100 à 1 à une distance du batteur égale à la profondeur.

Il semble donc que l'on puisse énoncer la règle pratique suivante : pour un batteur construit
« raisonnablement » mais sans précaution spéciale, une zone de longueur égale a trois fois la profondeur du canal doit suffire à la houle pour

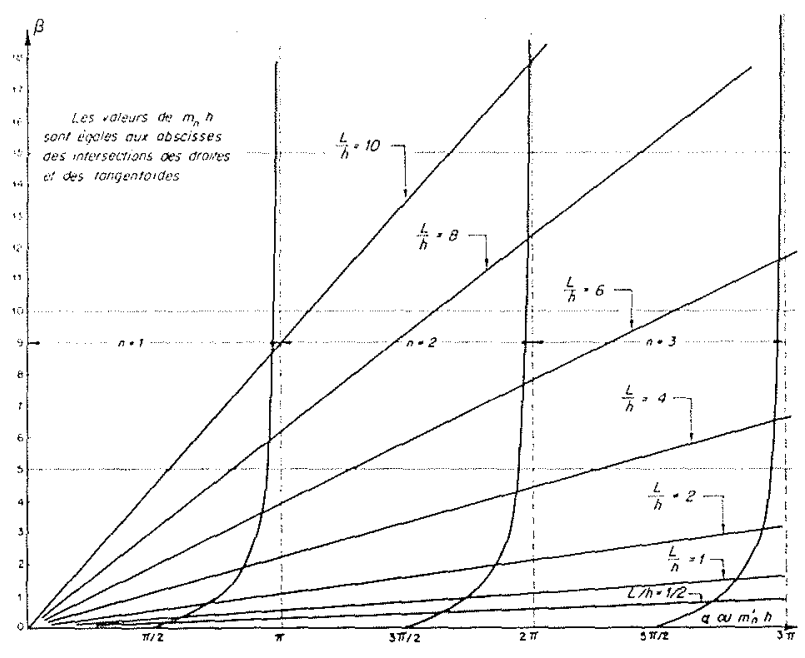

Fig. 4. - DÉteruiration GRapheue de valeurs DE $m_{n} h$ pOUR DIPFERENTES VALEURS DE L/h.

compenser naturellement le « défaut de forme " du mouvement du batteur.

$2^{\circ}$ Etude de oueloues cas particulitrs.

\section{A. - Battenr piston.}

Nous allons étudier tout d'abord un type de batteur particulièrement simple : le balteur à translation (voir figure 5) que nous appellerons aussi batteur piston.

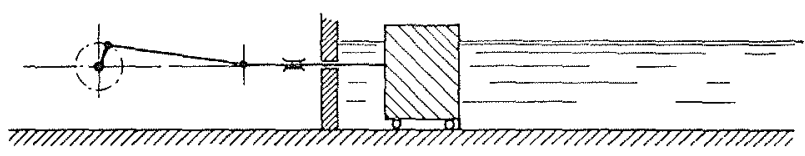

FIC. 5. - RePRESENTATON SCHBMATIQYe: D'D \& BATTEUR PISTON

Pour ce type de batteur la fonction $\xi(y)$ a la forme très simple : $\zeta(y)=\ell$

$e$ étant une constante.

La fonction $\xi(y)$ étant ainsi donnce, on calcule aisément, à l'aide de la formule (24), l'amplitude a de la houle produite (1) il vient:

(1) Dans ce qui suit, nous désignerons par amplitude d'un mourement sinusoïal l'élongation maximum du mouvement et non le double de cette quantité comme on le fait parfois. 


$$
\begin{aligned}
a & =c \operatorname{sh} m h=\frac{2 m h \int_{0}^{h} e \operatorname{ch} m \alpha d \alpha}{\operatorname{sh} m \operatorname{ch} m h+m h} \operatorname{sh} m \\
& =\frac{2 \operatorname{sh} 2 m h}{\operatorname{sh} m h \operatorname{ch} m h+m h} \quad e=\mathrm{K} e
\end{aligned}
$$

en posanl :

$$
\mathrm{K}=\frac{2 \operatorname{sh}^{2} m h}{\operatorname{sh} m h \operatorname{ch} m h+m h}
$$

La variation $d u$ coefficient $K$ en fonction $d u$ rapport $\mathrm{L} / h$ est donnée par le graphique de la figure 6 . On voit que pour les houles très courtes lamplitude est environ le double de celle du volet alors que pour les houles les plus longues le rapport $\mathrm{K}$ tend vers zéro.

On voit done quindépendamment de toute perte d'énergie l'amplitude des houles produites varie lorsque l'on fait varier la fréquence du mouvement du batteur sans modifier son amplitude. Si on désire étudier plusieurs houles de mêmes amplitudes mais de fréquences différentes, il est donc nécessaire de rajuster l'amplitude du batteur après chaque changement de vitesse.

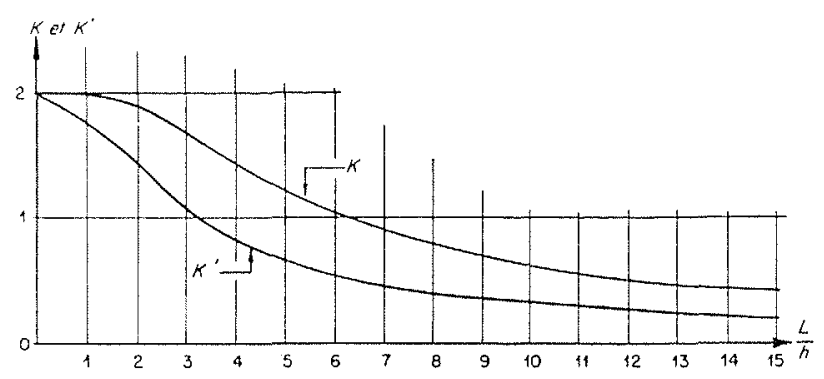

Fig. $6 .-$ Varuations des confricients $K$ et $K^{\prime}$ EN FONCTION DE $\mathrm{L} / h$.

Cette conclusion est d'ailleurs très générale et ne tient pas à la forme particulière du batteur étudié.

Considérons maintenant les mouvements parasites que nous avons qualifiés de transitoires dans l'espace. Afin d'abréger cette discussion tout en obtenant rapidement une idée de l'importance des phénomènes, nous ne considérerons, dans ce qui suit, que l'amplitude verticale des mouvements transitoires. Cette amplitude se déduit facilement de l'équation (23); dans le cas présent, elle est donnée par la formule :

$\sum_{n=1}^{\infty} 2 e \frac{\sin m_{n} h}{\sin m_{n} h \cos m_{n} h+m_{n} h} \sin m_{n} y e^{-m_{n} x} \sin k t$

Il suffira de considérer ces élongations au temps $\mathrm{T} / 4(\boldsymbol{k} t=\pi / 2)$ où elles passent par un maximum correspondant à la position la plus avancée du batteur. Laissons également provisoirement de côté les lacteurs $e^{-m_{n^{i}}}$, ce qui revient à considérer tout d'abord les amplitudes au droit du volet.

On peut distinguer pour chaque composante de rang $n$ :

Les amplitudes en surface (variation de niveaul :

$r_{i n}=c_{2} \sin m_{n} h=2 e \frac{\sin ^{2} m_{n} h}{\sin m_{n} h \cos m_{n} h+m_{n} h}$

et les amplitudes maxima :

$$
c_{n}=2 e \frac{\sin m_{n} h}{\sin m_{n} h \cos m_{n} h+m_{n} h}
$$

Nous allons faire l'application de ces formules à quelques cas particuliers.

a) Batteur piston émettant des houles longues.

Soit à étudier des houles dont la longueur est egale à huit fois la profondeur $(\mathrm{L} / h=8)$. On calculera successivement, en se limitant aux trois premiers termes de la perturbation et en s'aidant de la figure 4 :

$$
\begin{aligned}
& m h=\frac{\pi}{4} \# 0,785 \\
& m_{1} h=\pi-0,173 \# 2,97 \\
& m_{2} h=2 \pi-0,082 \# 6,2 \\
& m_{3} h=3 \pi-0,055 \# 9,37
\end{aligned}
$$

et ensuite, grâce aux formules (28) et (29):

$$
\begin{array}{ll}
\eta_{11}=0,021 e & c_{1}=0,123 e \\
\eta_{2}=0,0022 e & c_{2}=-0,027 e \\
\eta_{3}=0,0006 e & c_{3}=0,012 e
\end{array}
$$

D'autre part, on peut calculer l'amplitude de la houle grâce à la formule (26); on trouve :

$$
a=0,785 e
$$

On voit donc que les perturbations du niveau moyen sont trìs faibles même au voisinage du batteur. L'amplitude verticale maximum des mouvements transitoires est atteinte au voisinage de la profondeur $h / 2$ et elle ne vaut environ que $0,11 \mathrm{e}$.

C'est-à-dire que, dans le cas choisi, elle est environ sept fois plus faible que celle de la houle.

On verrait sans difficulté que l'amplitude horizontale, qui est maximum au voisinage du fond

(Voir suile page 482.) 


\section{Tableau synoptique}

\section{des différents appareils générateurs de houle}

La première partie de l'article de M. Búses, publice dans notre numéro 2/1951, était précédée de quelque paģes de M. Svouer intitulées: "Caractéristiques et essai de classification des appareils générateurs de houle».

Dans cet avant-propos, l'auleur annoncait la publication, répartic tout au long de l'article de M. Búset, de fiches donnant, pour chacun des types dappareils envisagés, un certain nombre de renscignements sur sa construction, son pouvoir réféchissant, sa mobilite, ete, ainsi que sur son comportement dans le cadre de la théorie de M. Bnésen.

Les fiches n" 1 a 4 ont paru dans le numéro 2/1951; le lecteur trouvera dans les pages (qui suivent les fiches $n^{0.5} 5$ à 11 .

M. Suguet avait cru bon de présenter, en tête de ces fiches, un tableau synoptique; nous pensons faciliter la tâche de nos lecteurs en reproduisant ce tableau ci-dessous.

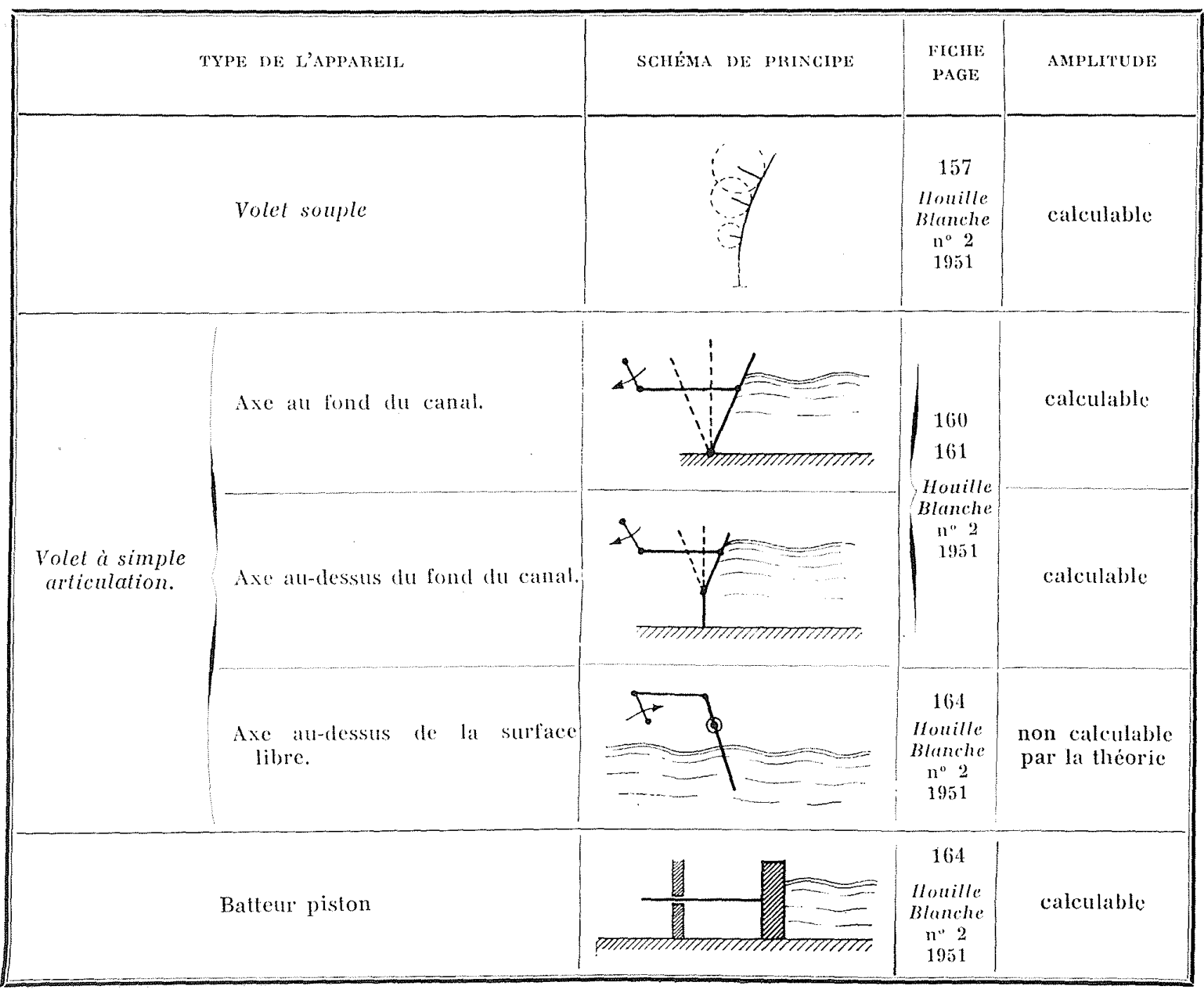




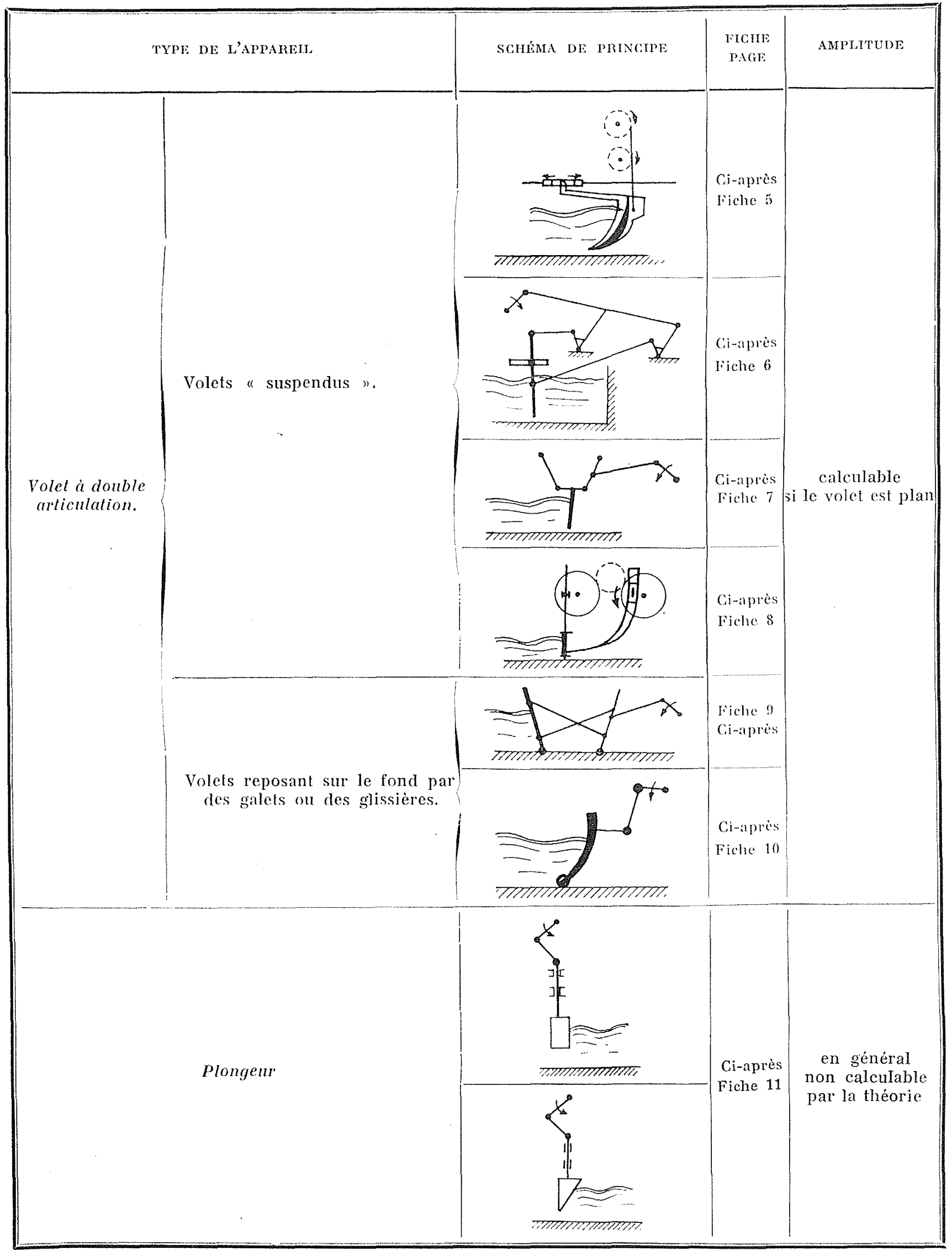




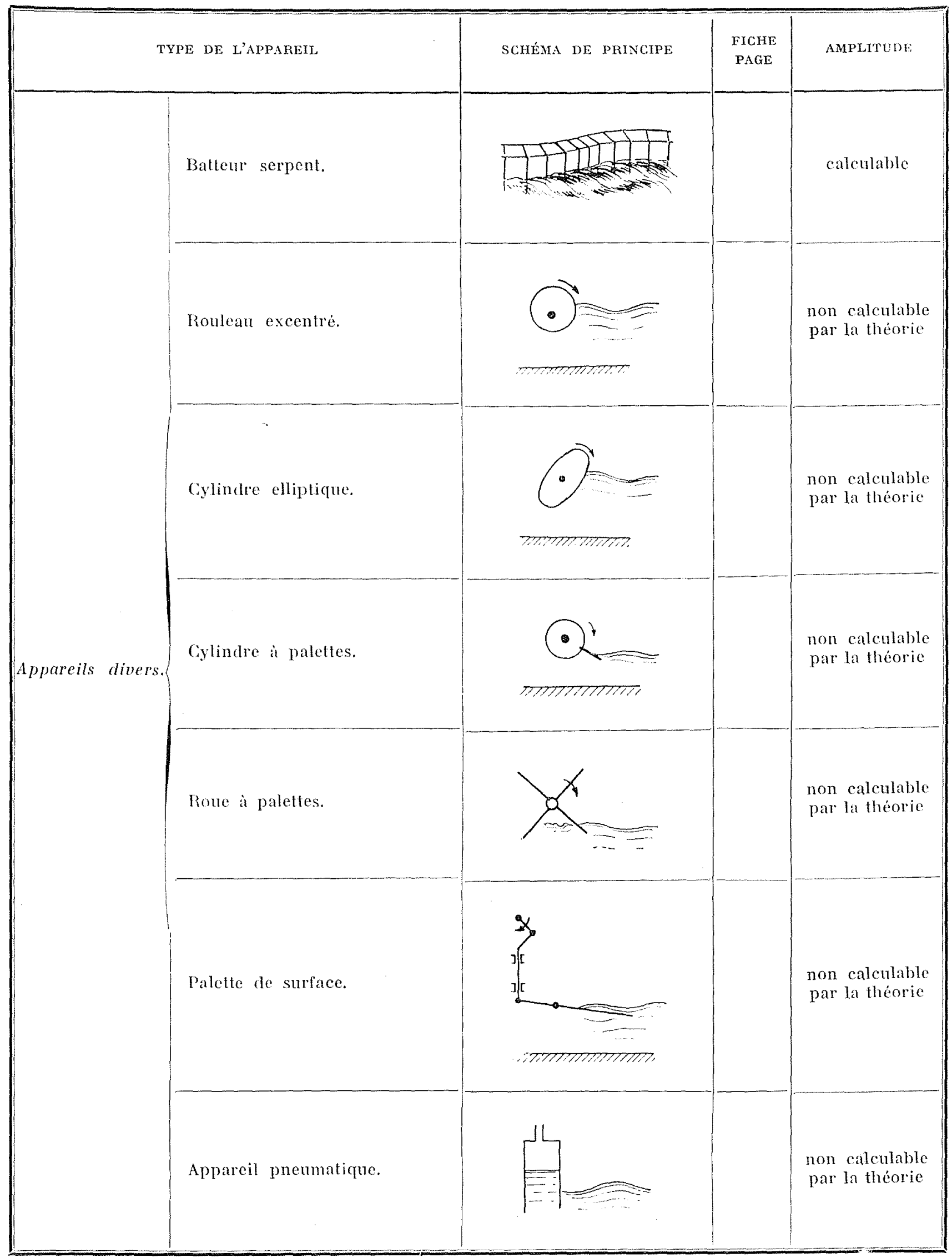




\section{FICHE No 5}

\section{Volet de la grande cuve à houle du bassin d'essais}

\section{des carènes de la Marine nationale - Paris}
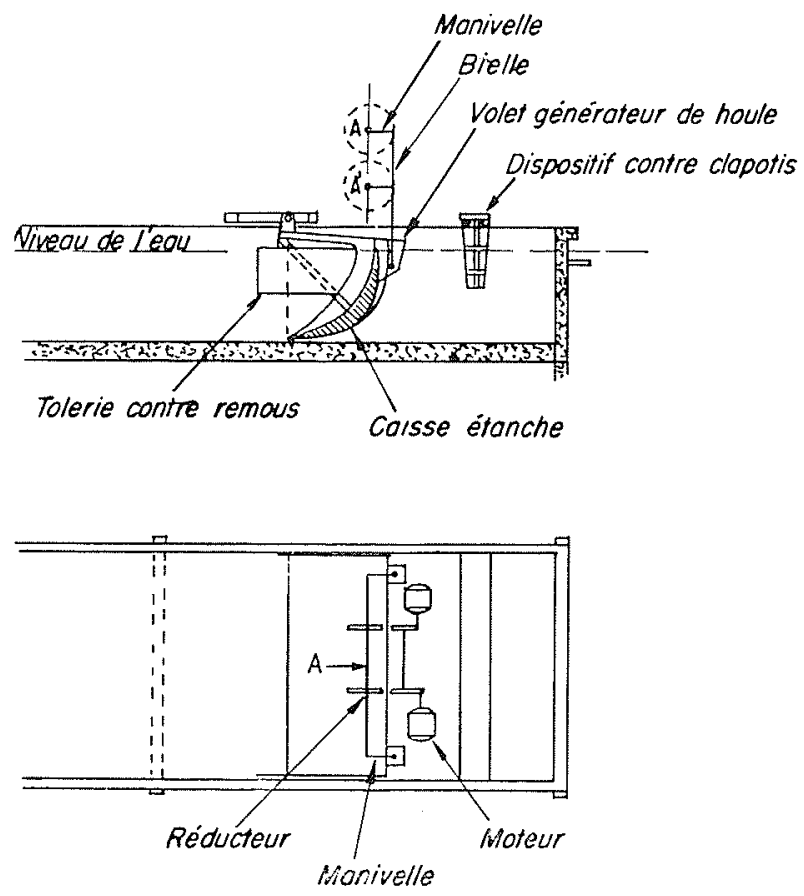

Ces dessins sont extraits du Rapport du Bassin d'Essais des Carènes pour l'année 1935

PRnNCIPE, - LOI DU MOUVEMENT,

C'est un volet courbe à double articulation; il repose sur deux glissières horizontales placées à la partie supérieure de la cuve et est mis en mouvement par deux bielles l'attaquant par la face arrière.

La forme du volet permet d'obtenir des trajectoires de particules liquides très proches de celles de la houle.

Calcul de l'amplitude:

L'amplitude ne peut être calculée d'après la theorie exposée dans cet article, car la forme du batteur, dans sa position moyenne, n'est pas un plan vertical.

Le réglage de l'amplitude se fait en modifiant la course des bielles.

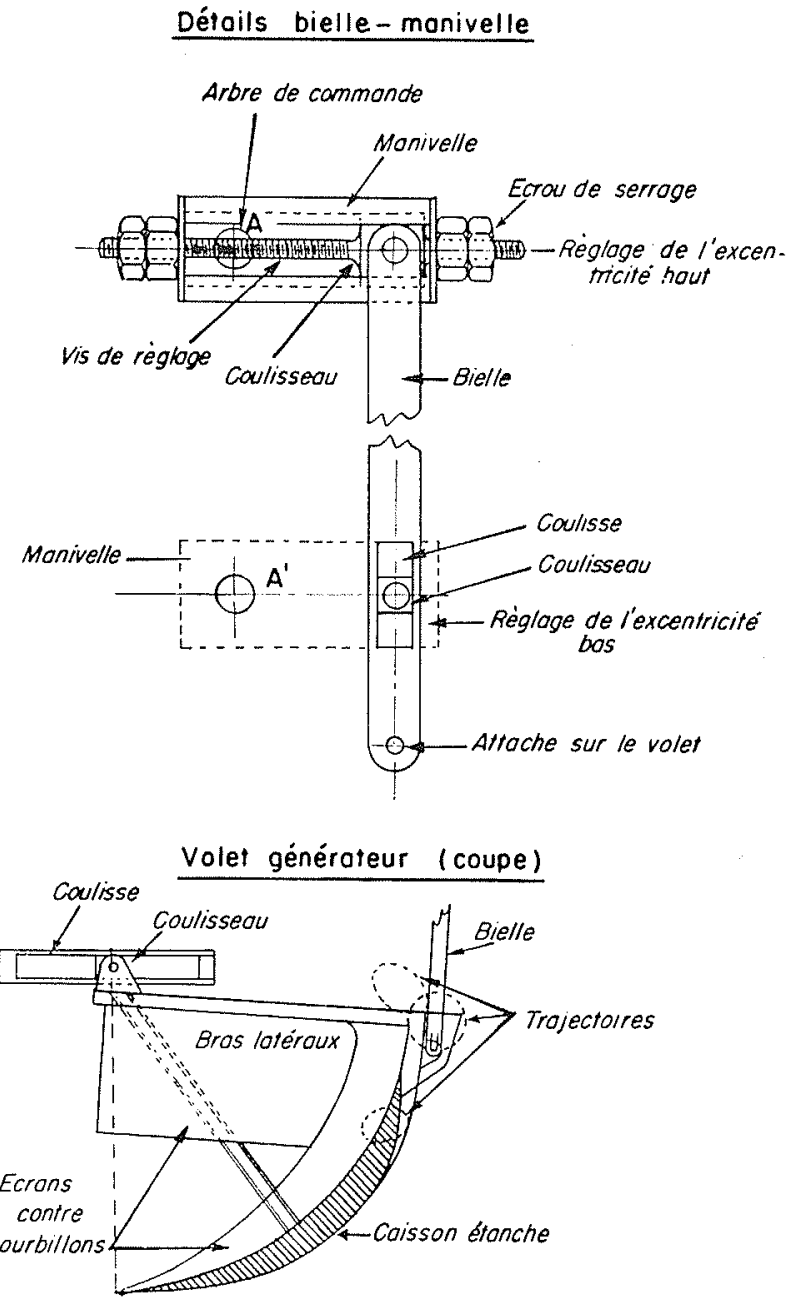

Remarque. - Ce batteur donne une houle très pure près du batteur et de longueur d'onde qui peut être de l'ordre de la longueur de la cuve. 
et de la surface, est du même ordre de grandeur. D'ailleurs cette amplitude horizontale n'est autre que la différence entre les amplitudes du batteur utilisé et celles du batteur parfait créant la même houle.

Dans le cas que nous venons d'étudier, non selilement la perturbation a des amplitudes faibles mais, de plus, leur décroissance est rapide. La composante d'indice 1, par exemple, n'a plus que $5 \%$ de son amplitude à une distance du batteur égale à la profondeur. L'amplitude maximum, au lieu d'être de l'ordre de grandeur du $1 / 7$ de celle de la houle, n'y est donc plus que le 1/140. La décroissance des autres composantes est, bien entendu, encore plus rapide.

En fait il est compréhensible que ces perturbations soient faibles puisque la forme du balteur est bien adaptée au mouvement de la houle. On sait en effet que pour les ondes longues par rapport à la profondeur les mouvements de l'eau ont prescue la meme amplitude au fond et à la surface.

b) Batteur-piston émettant des houles courtes.

Il est logique de s'attendre à ce que la conclusion que tious venons d'énoncer soit modifiée dans le cas des houles très courtes. Etudions par exemple le cas d'une houle dont la longueur est égale à la moitié de la profondeur du canal $(\mathrm{L} / h=1 / 2)$, on calcule comme précédemment, en s'aidant de la figure 4 :

$$
\begin{aligned}
& m h=4 \pi \\
& m_{1} h=\frac{\pi}{2}+0,135 \# 1,7
\end{aligned}
$$

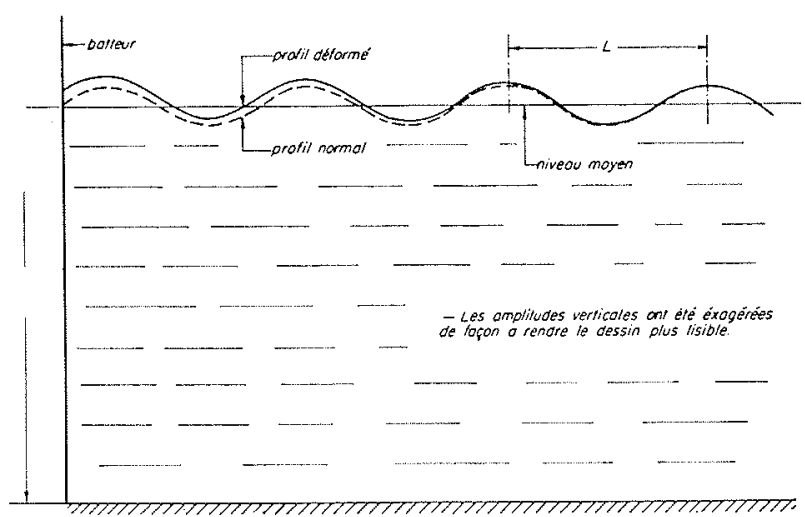

Fig. 7. - Batteur piston a smere transiation bMetcant UNE HOULE COURTE PAR RAPPORT A LA PROFONDEUR $\left(\mathrm{L} / h_{.}=1 / 2\right)$.

DEFORMATION DE LA HOULE AU VOISINAGE DU BatTEUR.

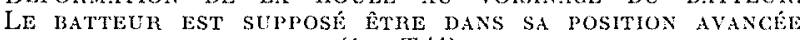
$(t=\mathrm{T} / 4)$.

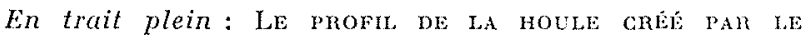
BATTEUR.

En trait interromples: Profle Nomal de Ia houle.

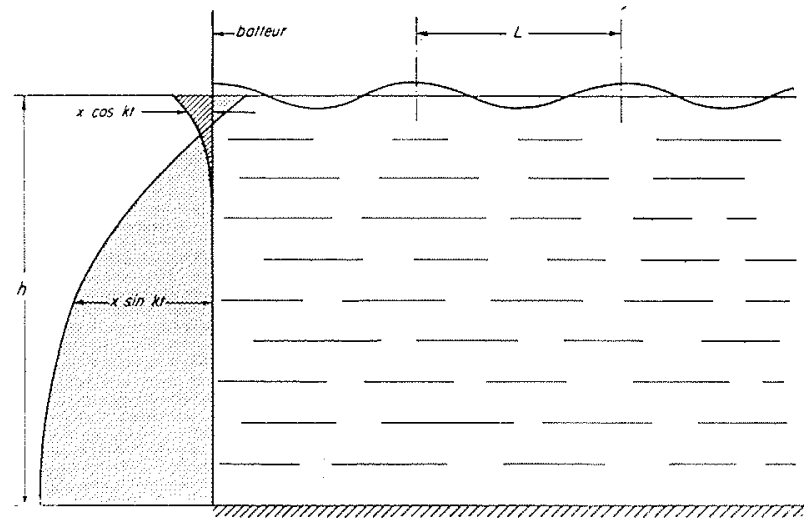

Fic. 8. - Batreur piston a smpte translation EMETTANT UNE HOULE COURTE PAR RAPPORT A LA PROFONDEUR $(\mathrm{L} / h=1 / 2)$.

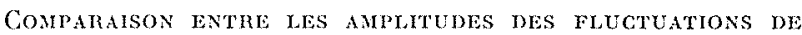
PRESSION NORMALES DUES A ha FOULE (AIRE HACHURÉE) ET LES FlUCTUATIONS DE PRESSION D'INERTIE (AIRE EN GRISÉ). N.B. - Le profil de la houle dont les amplitudes ont été exagérées est dessiné pour $\mathrm{t}=0$.

$$
\begin{aligned}
& m_{0} h=\frac{3 \pi}{2}+0,386 \# 5,1 \\
& m_{3} h=\frac{5 \pi}{2}+0,592 \# 8,45
\end{aligned}
$$

et ensuite on en déduit :

$$
\begin{array}{ll}
r_{11}=1,25 e & c_{1}=1,26 e \\
r_{12}=0,362 e & c_{2}=-0,39 e \\
r_{3}=0,172 e & c_{3}=0,207 e
\end{array}
$$

et $a \# 2 e$

La figure 7 montre l'allure des déformations apportées au profil d'onde au voisinage du batteur. On voit que ces deformations ne sont pas négligeables par rapport à l'amplitude de la houle. Comme elles changent de signe quand le batteur est dans sa position opposée et que la houle a alors avancé d'une demi-longueur d'onde, on peut voir que l'amplitude apparente de la houle est augmentée à des distances du batteur égales à $1 / 4,5 / 4,9 / 4$ d'onde, etc., et au contraire diminuée à des distances égales à $3 / 4,7 / 4,11 / 4$ d'onde, ete... Cette remarque montre que si l'on cherche à déceler des réftexions (clapotis partiel) par des mesures différentielles d'amplitude, il ne faut pas se placer trop près du batteur.

Terminons l'étude de ce cas par le calcul des pressions le long du volet.

La pression hydrostatique sera :

$$
p_{\mathrm{II}}=g(h-y)
$$




\section{FICHE N॰6}

\section{Volet \\ du "Laboratoriet for Havnebygning of Fundering" Copenhague}

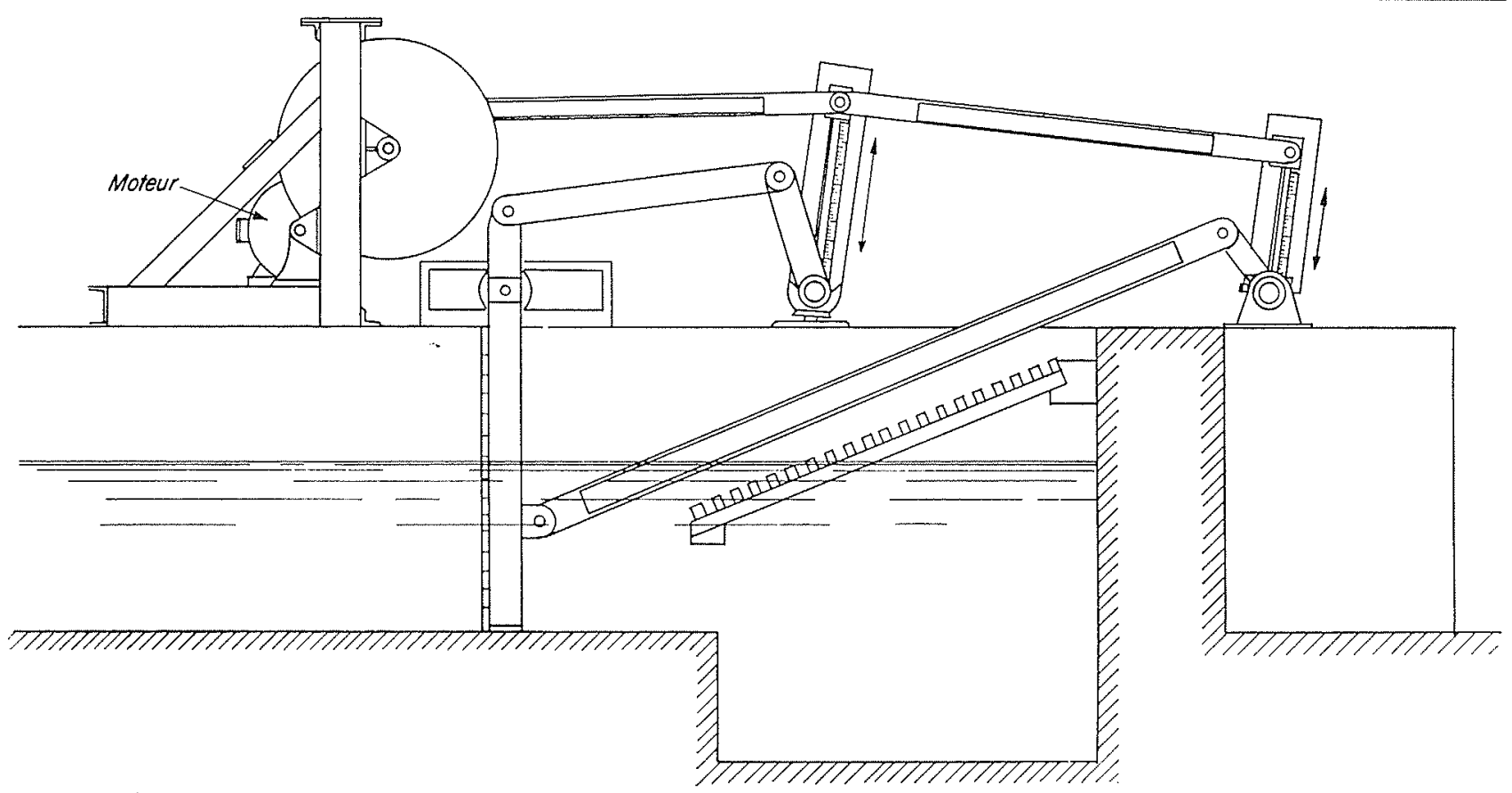

Communiqué par le Laboratoriet for Havnebygning of Fundering - Danmarks Tekniske Hoyskole

Principe. - LoI DU Mouvement.

C'est un volet plan à double articulation; il repose sur des glissières placées à la partie supérieure du canal et est actionné par un système de bielles qui l'attaquent par la face arrière.

Calcul de l'amplitede de la houle produte:

Le volet étant constitué par un plan, vertical dans sa position moyenne, la théorie de M. Bresel permet de calculer l'amplitude : il suffit de superposer

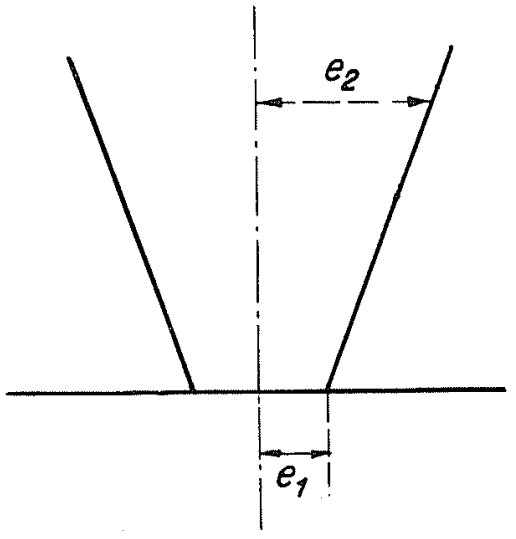

les solutions obtenues pour le batteur à simple articulation et le batteur piston. On obtient ainsi pour la demi-amplitude, avec les notations de la figure

$$
\begin{gathered}
a=2\left(e_{2}-e_{1}\right) \frac{(1-\operatorname{ch} m h+m h \operatorname{sh} m h) \operatorname{sh} m h}{(\operatorname{sh} m h \operatorname{ch} m h+m h) m h} \\
+2 e_{1} \frac{\operatorname{sh}^{2} m h}{\operatorname{sh} m h \operatorname{ch} m h+m h}
\end{gathered}
$$

\section{INERTIE :}

L'inertie n'est pas plus grande que celle du batteur à simple articulation.

\section{Entretren :}

Il n'y a qu'une articulation susceptible de plonger dans l'eau; l'entretien est donc très faible.

POUVOIR RÉFLÉCHISSAN'T :

Il a un coefficient de réflexion voisin de celui du volet à simple articulation.

\section{RÉGLAGE :}

Grâce au système de bielles, il est possible de modifier commodément l'amplitude ainsi que le mouvement au fond du canal sans arrêter le batteur. 
La fluctuation de pression normale due à la houle sera :

$$
p_{n}=\operatorname{sga} \frac{\operatorname{ch} m y}{\operatorname{ch} m h} \cos k t
$$

Soit, dans le cas présent :

$$
p_{n}=2 e . g \frac{\operatorname{ch} m y}{\operatorname{ch} m h} \cos k t
$$

La fluctuation de pression d'inertie vaut :

$$
p_{i}=\circ g \sum_{n=1}^{\infty} c_{n} \operatorname{tg} m_{n} h \cos m_{n} y \sin k t
$$

Si nous nous limitons aux trois premiers termes, on a, dans le cas présent :

$$
\begin{aligned}
p_{i}= & -\left(9,3 \cos 1,7 \frac{y}{h}-0,96 \cos 5,1 \frac{y}{h}\right. \\
& \left.+0,31 \cos 8,45 \frac{y}{h}\right) \circ g e \sin k t
\end{aligned}
$$

Au niveau du fond, par exemple, la fluctuation a pour amplitude - $8,65 \circ g e$, soit plus de quatre fois l'amplitude maximum de la fluctuation de pression due à la houle. La figure 8 permel d'ailleurs de se rendre compte de l'importance relative des deux fluctuations de pression. On voil que la fluctuation d'inertie, trop souvent négligée dans les calculs de batteurs, peut ètre considérable.

Si l'on calcule la charge maximum totale subie par le batteur du fait de ces différentes fluctuations, on trouve :

Pour la pression normale :

$$
\mathrm{P}_{n}=\int_{u}^{h} p_{n} d y=\frac{2 e \rho g}{m} \text { th } m h
$$

Pour la pression d'inertie :

$$
\begin{aligned}
\mathrm{P}_{i}=\int_{0}^{h} p_{i} d y= & \varsigma g \text { eh }\left[\frac{9,3}{1,7} \sin 1,7-\frac{0,96}{5,1} \sin 5,1\right. \\
& \left.+\frac{0,31}{8,45} \sin 8,45\right] \\
& =5,6 \% g \mathrm{eh}
\end{aligned}
$$

Le rapport $\frac{\mathrm{P}_{i}}{\mathrm{P}_{n}}$ vaut :

$$
\frac{\mathrm{P}_{i}}{\mathrm{P}_{n}}=\frac{5,6 \circ g e m h}{2 \circ g \mathrm{eth} m} \# 35
$$

La charge totale maximum due à l'inertie vaut done environ trente-cing fois la charge totale maximum déduite des équations usuelles de la houle.

Ces résultats sont essentiels en ce qui concerne le calcul de la résistance des appareils producteurs de houle et de leurs mécanismes, mais ils sont également importants pour l'étude de l'étanchéité du batteur. En eff'et, on se base souvent sur le fait que la fluctuation de pression due aux houles (pression normale) décroît très rapidement avec la profondeur pour se dispenser complètement de mettre des étanchéités à la base du batteur, voire même laisser la base de co dernier à quelque distance au-dessus du fond du canal. Nous venons de montrer que les fluctuations de pression peuvent rester très importantes en profondeur, par conséquent ce défaut d'étanchéité se traduit par des pertes d'énergie, un accroissement de la turbulence au droit du batteur et surtout une déformation des houles produites.

Ne terminons pas sans souligner que l'importance considérable des fluctuations de pression d'inertie est due à ce que l'exemple que nous venons d'étudier est typiquement défavorable. Il est en effet anormal d'émettre une houle aussi courte avec un batteur dont le mouvement est spécialement bien adapté aux houles longues.

\section{B. - Volet battant.}

Nous allons considérer maintenant un autre type de batteur qui est extrêmement répandu parce que trìs facile à construire. Il s'agit du volet battant à simple articulation tel qu'il est représenté schématiquement sur la figure 9 :

Nous désignerons par e l'élongation maximum

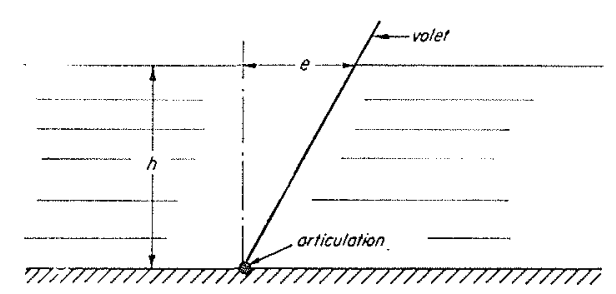

FIG. 9

du volet à la hauteur du niveau moyen. La fonetion $\zeta(y)$ sera done donnée par :

$$
\xi(y)={ }_{h}^{c} y
$$

Nous calculerons l'amplitude de la houle comme précédemment à l'aide de la formule (24). On a :

$$
\begin{gathered}
a=\frac{2 a \int_{\|}^{h} \frac{e}{h} \alpha \operatorname{ch} m \alpha d \alpha}{\operatorname{sh} m h \operatorname{ch} m h+m h} \operatorname{sh} m h \\
=2 \frac{1-\operatorname{ch} m h+m h \operatorname{sh} m h}{m h(\operatorname{sh} m h \operatorname{ch} m h+m h)} \operatorname{sh} m h e
\end{gathered}
$$

Soit $\quad a=\mathrm{K}^{\prime} e$

en posant :

$$
\mathrm{K}^{\prime}=2 \frac{\operatorname{sh} m h(1-\operatorname{ch} m h+m h \operatorname{sh} m h)}{m h(\operatorname{sh} m h \operatorname{ch} m h+m h)}
$$




\section{FICHE No 7}

\section{Volet "balançoire" Neyrpic}

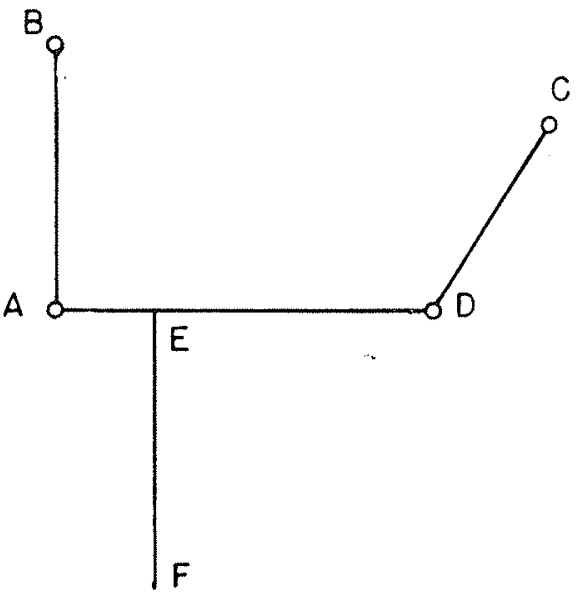

Photo et schéma

du Laboratoire Dauphinois d'Hydraulique Neyrpic

\section{Principe. - Loi du mouvement.}

C'est un volet plan à double articulation dont le principe de fonctionnement repose sur les propriétés du « trois barres ». Une description complète en a été faite par M. G. RANSFORD dans une communication du Congrès de l'A.I.R.T.H. de 1949 à Grenoble: "A wave machine of novel type."

Très schématiquement, il se compose de trois barres articulées entre elles en A et D : l'ensemble peut osciller autour des articulations fixes B et C. Le volet proprement dit est en EF.

\section{Calcul de l'ampltede de la houle phodute:}

Le volet étant un plan, vertical dans sa position moyenne, la théorie de M. Bresel, exposée dans cet article, permet de calculer l'amplitude de la houle produite; il sulfit de superposer les solutions obtenues pour le batteur à simple articulation et le batteur-piston. On obtient ainsi pour demi-amplitude:

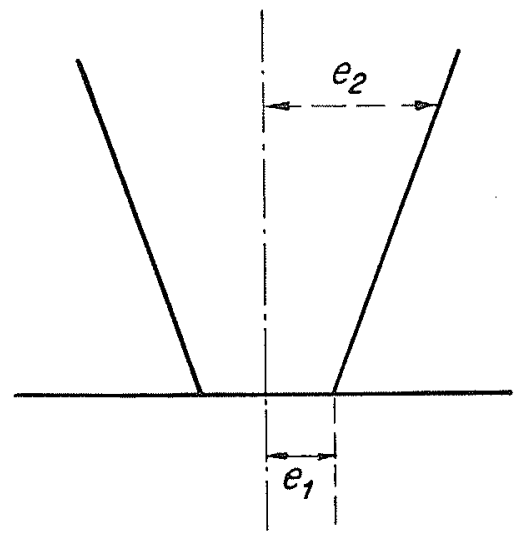

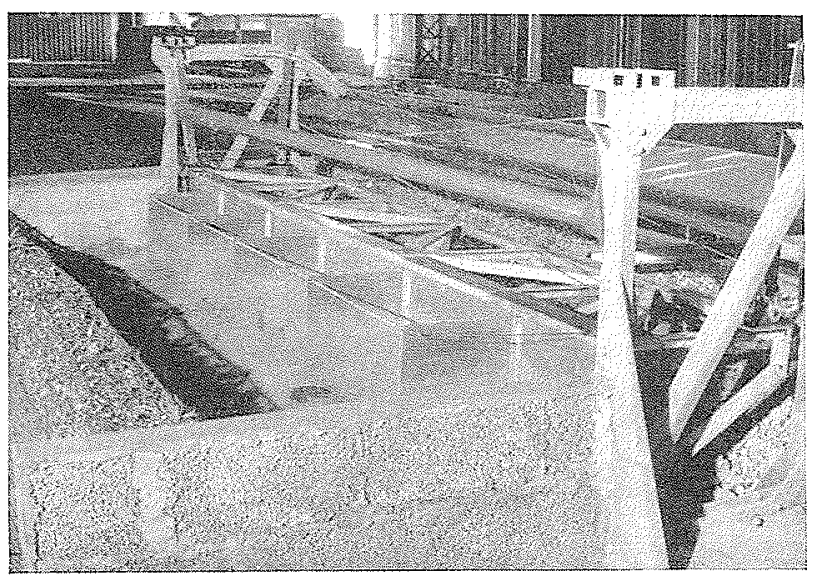

$$
\begin{gathered}
a=2\left(e_{2}-e_{1}\right) \frac{(1-\operatorname{ch} m h+m h \operatorname{sh} m h) \operatorname{sh} m h}{(\operatorname{sh} m h \operatorname{ch} m h+m h) m h} \\
+2 e_{1} \frac{\operatorname{sh}^{2} m h}{\operatorname{sh} m h \operatorname{ch} m h+m h}
\end{gathered}
$$

\section{Construction :}

Elle est relativement très simple. On peut toutefois être gêné par la question de l'étanchéité sous le balteur.

\section{INERTIE :}

Elle n'est pas plus grande que celle du batteur à simple articulation.

\section{ENTRETIEN :}

L'appareil est très robuste et son entretien minime, du fait qu'il n'existe aucune articulation on galet sous l'eau.

\section{POUVOTR RÉFLÉCHISSANT:}

Le batteur est réfléchissant et peut nécessiter l'emploi d'un filtre.

\section{RÉGLAGE :}

Le réglage de l'amplitude se fait en modifiant l'excentricié de la manivelle; le réglage de la course du volet au fond du canal se fait en modifiant la position du point d'articulation $\mathrm{C}$.

\section{MOBILI'TÉ :}

Quoique ses qualités de mobilité soient inférieures à celles du volet à simple articulation, il est relativement facile de le faire fonctionner avec des orientations différentes au moyen d'un montage approprié. 
La variation de $\mathrm{K}^{\prime}$ en fonction du rapport $\mathrm{L} / h$ est indiquée sur le graphique de la figure 6.

Pour une même élongation en surface, on voit que le volet battant fait toujours des houles d'amplitude plus faible que le volet piston, en particulier, pour les grandes longueurs d'onde, le rapport des amplitudes est environ de 1 à 2 . Cependant, pour les très petites longueurs d'onde, la différence entre $K$ et $K^{\prime}$ tend à disparaìtre.

Calculons maintenant les perturbations transitoires dans l'espace. Comme précédemment nous nous bornerons aux trois premiers termes du développement et à l'amplitude verticale.

Cette amplitude se déduira facilement de l'équation (23) et clle sera dans le cas présent :

$\sum_{n=1}^{\infty} 2 e \frac{m_{n} h \sin m_{n} h+\cos m_{n} h-1}{m_{n h} h\left(\sin m_{n} h \cos m_{n} h+m_{n} h\right)} \sin m_{n} y e^{-m_{n} x} \sin k t$

On en déduit comme précédemment pour chaque composante :

Les amplitudes en surface (variation de niveau) :

$n_{n}==c_{n} \sin m_{n} h=2 e \frac{\sin m_{n} h\left(m_{n} h \sin m_{n} h+\cos m_{n} h-1\right)}{m_{n} h\left(\sin m_{n} h \cos m_{n} h+m_{n} h\right)}$

et les amplitudes maximum :

$$
c_{n}=2 e \frac{m_{n} h \sin m_{n} h+\cos m_{n} h-1}{m_{n} h\left(\sin m_{n} h \cos m_{n} h-1-m_{n} h\right)}
$$

Faisons lapplication de ces formules aux mêmes cas particuliers que précédemment :

a) Volet battant émettant des houles très longues $(\mathrm{L} / h=8)$, il vient:

$$
\begin{array}{ll}
n_{11}=-0,061 e & c_{1}=-0,365 e \\
n_{2}=0,0022 e & c_{2}=-0,027 e \\
n_{3}=-0,0019 e & c_{3}=-0,034 e
\end{array}
$$

et d'autre part on $\mathbf{a}$ :

$$
a=0,408 e
$$

On voit que pour une même élongation du volet en surface le batteur étudié ne crée qu'une houle sensiblement moitié de celle obtenue avec le batteur piston. D'autre part, les perturbations transitoires, presque nulles dans le cas du batteur piston, deviennent très sensibles. Si nous ne considérons que les déformations de la surface libre au voisinage du volet, nous voyons que celles-ci sont de l'ordre de $15 \%$ de l'amplitude de la houle. L'importance des perturbations transitoires apparait encore plus clairement si l'on considère l'élongation verticale maximum qui est atteinte au voisinage de la profondeur $h / 2$ et qui vaut environ $0,36 e$, c'est-à-dire presque $90 \%$ de l'amplitude de la houle.
Ces résultats ne sont pas étonnants puisque le batteur que nous venons d'étudier diffère beaucoup plus du batteur idéal pour ondes longues que le batteur piston. Cependant, là encore, nous observerons une décroissance très rapide des perturbations et à une distance du batteur égale à trois profondeurs elles seront toutes complètement négligeables.

b) Volet battant émettant des houles très courtes.

Nous reprenons le cas déjà traité pour le batteur piston $(\mathrm{L} / h=1 / 2)$.

On a alors :

$$
\begin{array}{rlrl}
\eta_{1} & =0,41 e & c_{1} & =0,413 e \\
\eta_{2} & =0,412 e & c_{2} & =-0,443 e \\
r_{13} & =0,133 e & c_{3} & =0,161 e \\
a=1,84 e &
\end{array}
$$

La comparaison avec les formules de la page 482 montre que, pour une amplitude de houle sensiblement identique, les déformations transitoires de surface sont deux fois plus faibles au voisinage immédiat du batteur et environ trois fois plus faibles à quelque distance, dès que les perturbations d'ordre supérieur au premier cessent de se faire sentir.

rTerminons là encore par le calcul des pressions le long du volet. On aura :

$$
p_{n}=1,84 e_{\rho} g \frac{\operatorname{ch} m y}{\operatorname{ch} m h} \cos k t
$$

$p_{i}=\rho g \Sigma c_{n} \operatorname{tg} m_{n} h \cos m_{n} y \sin k t$

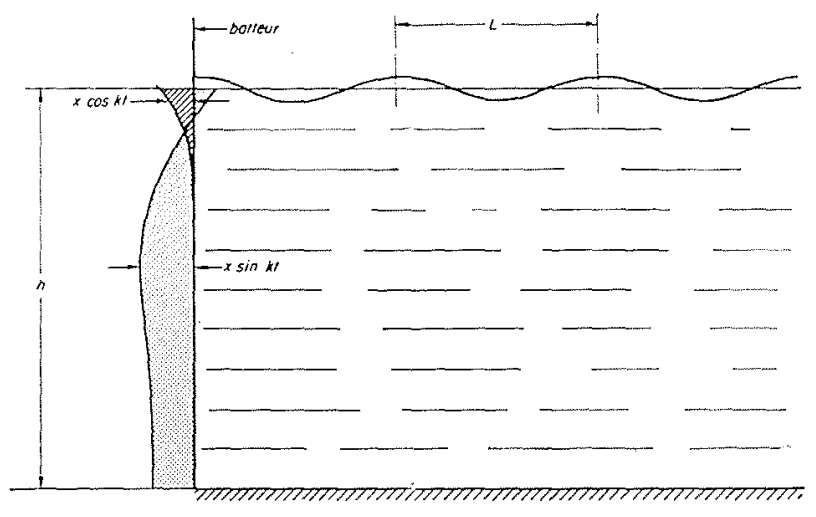

Fig. 10. - Battevr volet a smple articulation EMETTAN' UNE HOULE COURTE PAR MAPPORT a ra ProfondeUR $(\mathrm{L} / h=1 / 2)$.

Comparaison entre les amplitudes des fluctuatons de PRESSION NORMALES DUES A LA HOULE (AIRE HACHURÉ) ET LES FLUGTUATIONS DE PRESSION D'INERTIE (AIRE EN GRISE), N.B. - Le profil de la houle, dont les amplitudes ont été exagérées, est dessiné pour $\mathrm{t}=0$. 
Soit :

$$
\begin{aligned}
p_{i}=-\operatorname{e\rho g}[ & 3,03 \cos 1,7 \frac{y}{h}-1,11 \cos 5,1 \frac{y}{h} \\
& \left.+0,24 \cos 8,45 \frac{y}{h}\right]
\end{aligned}
$$

Nous voyons done que les fluctuations de pression d'inertic, tout en restant dans leur ensemble nettement plus importantes que les fluctuations de pression normale, sont environ trois fois plus faibles que dans le cas du volet piston. Les figures 8 et 10 permettent de comparer commodément les fluctuations de pression accompagnant le fonctionnement de ces deux types de batteur.

Intégrons comme précédemment pour trouver la charge tolale maximum due à ces différentes fluctuations de pression, il vient :

$$
\mathrm{P}_{n}=1,84 \frac{e \rho g}{m} \text { th } m h
$$

$$
\begin{gathered}
\mathrm{P}_{i}=\rho g \operatorname{eh}\left[\frac{3,03}{1,7} \sin 1,7-\frac{1,11}{5,1} \sin 5,1\right. \\
\left.+\frac{0,24}{8,45} \sin 8,45\right]=2 \circ g \mathrm{eh}
\end{gathered}
$$

le rapport $\frac{\mathrm{P}_{i}}{\mathrm{P}_{n}}$ vaut done :

$$
\frac{\mathrm{P}_{i}}{\mathrm{P}_{h}}=\frac{2 \rho g e \mathrm{mh}}{1,84 \rho g e \operatorname{th} m h}=13,6
$$

alors que ce rapport étail de 35 dans le cas du batteur piston.

Nous arrêtons là l'étude des cas particuliers pensant que ces quelques exemples ont suffi à montrer le fonctionnement et l'utilité de la théorie.

\section{IV. - Conclusions générales}

Dans ce qui suit, nous voudrions faire le point des résultats qui se dégagent des calculs précédents. Nous chercherons à mettre en relief d'une part l'intérêt pratique que les résultats obtenus peuvent présenter pour la conception des batteurs à houle, et d'autre part les insulfisances de la théorie, en indiquant sur quels points il faut chercher à obtenir des précisions nouvelles.

Le résultat fondamental de ces calculs est de montrer que le «batteur idćal » défini, rappelons-le, à partir de théories du premier ordre, n'a pas le caractère privilégié qu'on a tendance à lui attribuer intuitivement. Les exemples choisis nous ont montré par exemple que, même dans le cas de batteurs très mal utilisés, - un batteur piston employé pour engendrer une houle tres courte ou un volet à simple articulation pour des houles très longues, - les perturbations à l'origine n'étaient que de l'ordre de grandeur de la houle créée, et que, de plus, ces perturbations étaient négligeables à une distance du batteur égale à trois fois la profondeur étant donné qu'à cette distance elles ne conservent tout au plus que le centième de leur amplitude initiale. Or il est rare que l'on ne puisse disposer d'une telle longueur devant le batteur (1).

Cette conclusion suppose bien entendu que le batteur construit ne soit pas trop déraisonnable (2). Si l'on s'attachait au contraire à cons-

(1) Espace qui d'ailleurs peut être utilisé pour mettre des filtres à houle.

(2) Nous nous excusons de l'imprécision de ce terme un peu trop subjectif, mais pratique et dont le sens est, nous semble-t-il, assez clair étant donné le contexte. truire un batteur paradoxal, comme par exemple un volet inversé ayant son articulation au niveau de l'eau ou mème un peu au-dessous, on pourrait avoir des perturbations initiales beaucoup plus grandes. On peut même montrer que pour chaque fréquence, il existe une position de l'axe d'un tel batteur pour laquelle il est incapable d'émettre de la houle. Son action se limite donc à créer la perturbation initiale. Il est d'ailleurs intéressant de noter qu'il y a une possibilité théorique de faire varier périodiquement le niveau à l'extrémité d'un canal sans que cette perturbation se transmette à l'autre extrémité par unn phénomène d'onde ou de seiche.

Sommes-nous donc fondés à conclure que tous les batteurs « raisonnables » sont également satisfaisants au point de vue hydraulique et que, par conséquent, le moins cher sera le meilleur?

Une telle conclusion, dont on conçoit l'importance économique, ne peut s'énoncer que sous une forme plus nuancée, car d'une part la théorie n'est pas parfaite, ainsi que nous l'avons déjà souligné, et d'autre part des considérations d'ordres très divers entrent en ligne de compte dans le choix d'un batteur. Nous voudrions, à ce propos, attirer l'attention du lecteur sur les points suivants :

$1^{\circ}$ En tout état de cause il sera nécessaire de rechercher un équilibre économique entre le prix du batteur et celui de la surlongueur qu'il pourra être nécessaire de donner au canal. En particulier si le bassin est large et profond et s'il doit être construit dans un bâtiment existant de di(Voir suite page 492.) 


\section{FICHE No 8}

\section{Volet du Laboratoire National d'Hydraulique-Chatou}

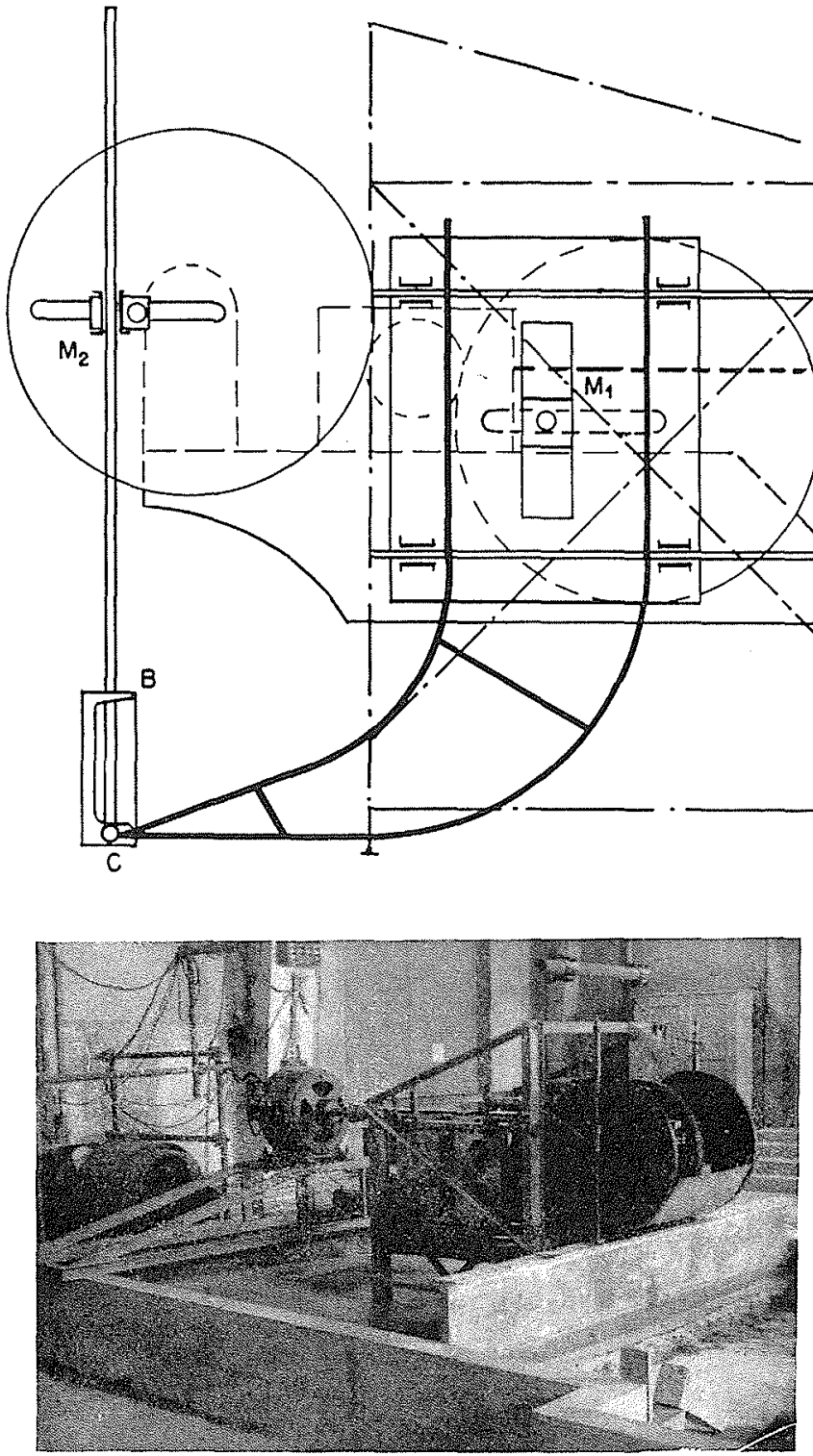

Principe. - Loi de mouvement.

C'est un volet plan à double articulation mis en mouvement par son extrémité inférieure reliée à la manivelle $M_{1}$ et par son extrémité supérieure reliée à la manivelle $M_{2}$. Les rotations de $M_{1}$ et $M_{2}$ sont synchronisées par un train d'engrenage de sorte qu'un point quelconque du batteur décrivant une ellipse, le point $\mathrm{C}$ décrive un segment de droite.

Schema photographies et renseignements communiques par le Laboratoire National d'Hydraulique

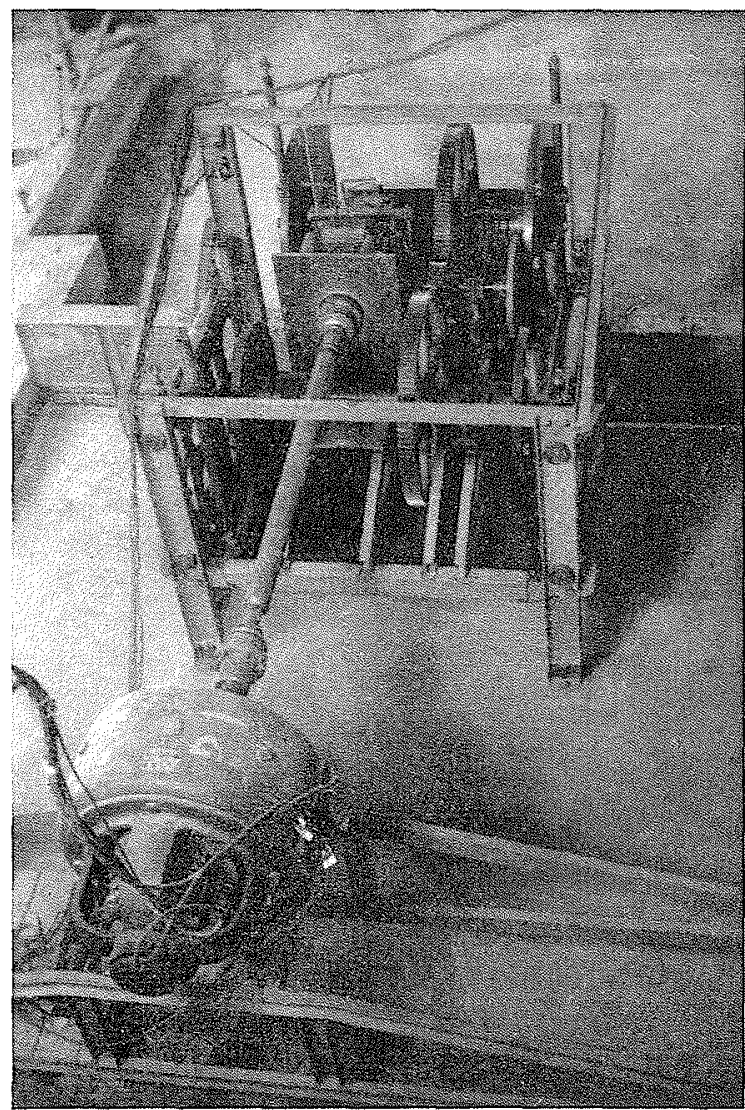




\section{FICHE No 8 (SUITE)}

Calcul de l'Amplitude de la houle produtte :

Le volet étant un plan, vertical dans sa position moyenne, la théorie de M. BIESEL, exposée dans cet article, permet de calculer l'amplitude de la houle produite; il suffit de superposer les solutions obtenues pour le batteur à simple articulation et le batteur piston. On obtient ainsi pour la demi-amplitude:

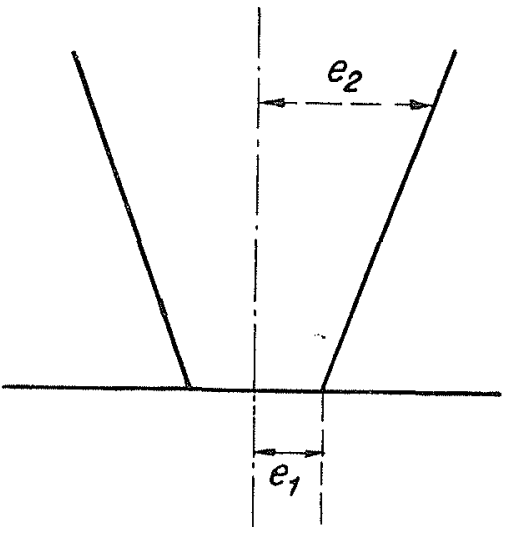

$a=2\left(e_{2}-e_{1}\right) \frac{(1-\operatorname{ch} m h+m h \operatorname{sh} m h) \operatorname{sh} m h}{(\operatorname{sh} m h \operatorname{ch} m h+m h) m h}$

$$
+2 e_{1} \frac{\operatorname{sh}^{2} m h}{\operatorname{sh} m h \operatorname{ch} m h+m h}
$$

\section{Entretien :}

Il existe le minimum d'articulations immergées.

PouvoIR RÉFLÉchissant :

Il a un coefficient de réflexion voisin de celui du volet plan à simple articulation.

RÉGLAGE :

Le mouvement des extrémités supérieure et inférieure se règle en agissant sur l'excentricité des manetons.

La vitesse de rotation des manivelles se règle au moyen d'une boite à quatre vitesses et d'un rhéostat; la période peut ainsi varier entre $1 / 3$ et 59 sec.

\section{MOBILTTÉ :}

Le Laboratoire National d'Hydraulique a étudié des éléments de batteur standard de 3 mètres de longueur susceptibles de fonctionner de façon synchrone. Chaque élément peut être monté sur un support indépendant du fond du modèle qu'on pent orienter aisément de facon à obtenir la direction de propagation voulue. 


\section{FICHE No 9}

\section{Volet du Laboratoire de l'Imperial College of Science and Technology de Londres Volet du Laboratoire d'Hydraulique de I'Université de Padoue}
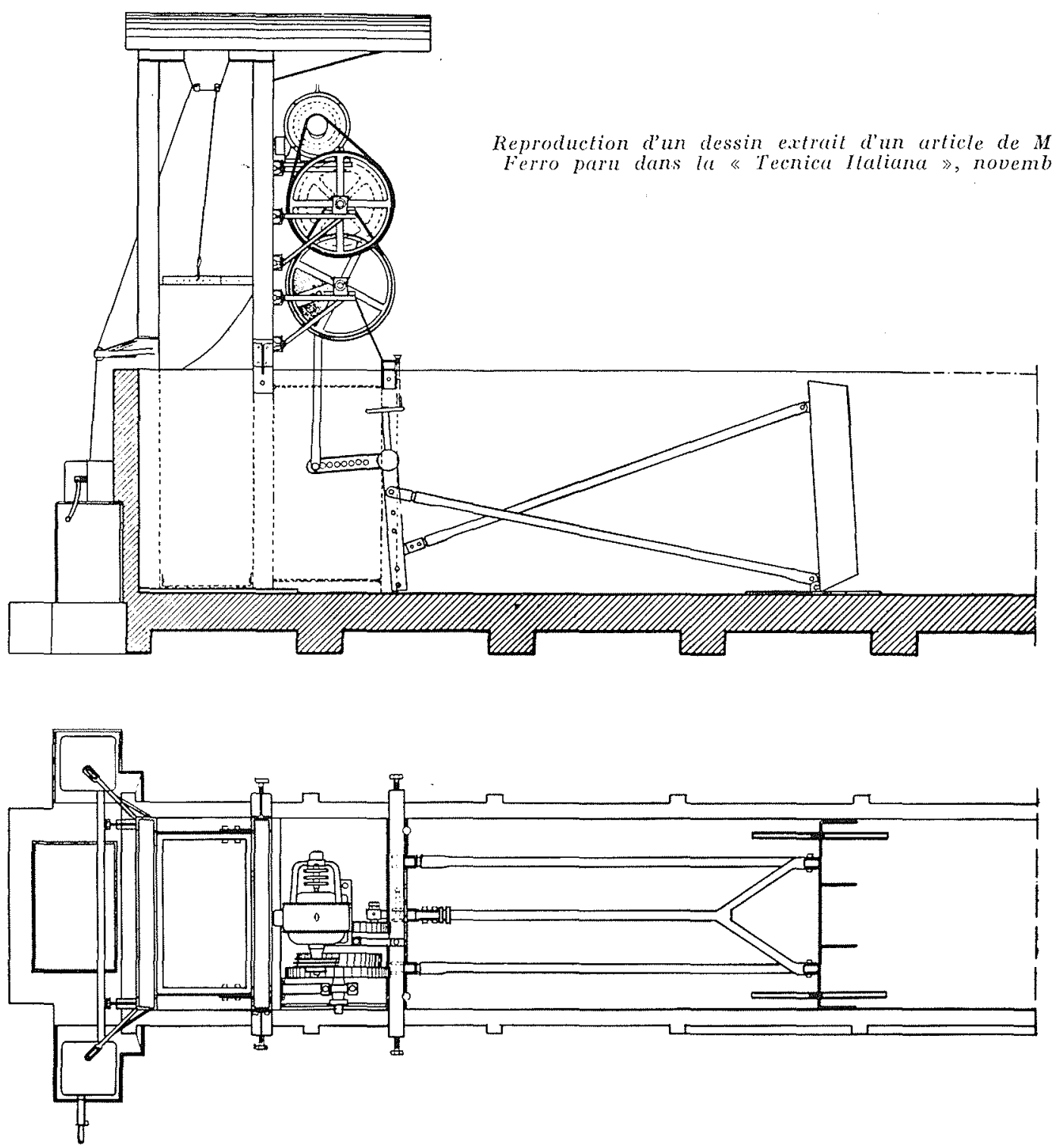

Nous avons porté ces deux volets sur la même fiche car leur principe est le même; seule diffère la facon dont est produit le mouvement : servo-motenr hydraulique dans le premier, motenr électrique dans le deuxième.
Princlee. - Loi du movvement.

C'est un volet plan à double articulation, attaqué par deux bielles articulées, lune a la partie supéricure, l'autre à la partie inférieure. Ces deux 


\section{FICHE No 9 (SUITE)}

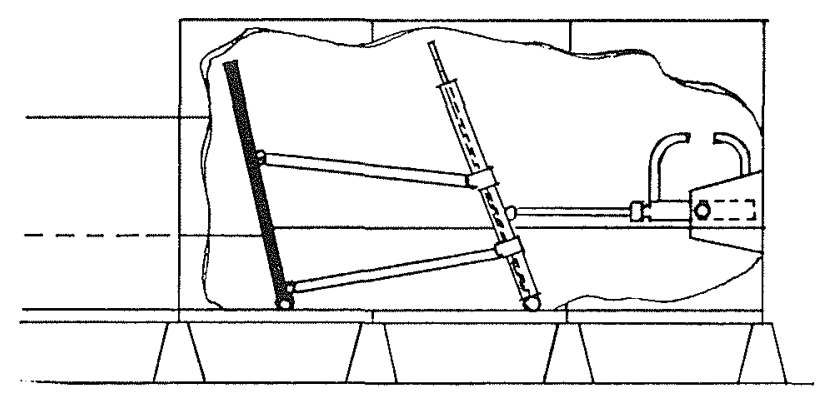

Reproduction d'un dessin extrait d'une communication du Brigadier R. A. Bagnold da «'Institution of Civil Engineers 》 1939

bielles sont à leur autre extrémité articulées sur un système de bras solidaires pouvant osciller autour d'un axe placé au fond du canal. Ce système de bras est mis en mouvement par une bielle reliée au piston d'un servo-moteur à huile pour le premier volet, à un maneton entraîné par un moteur électrique pour le second.

Le volet comporte des galets à sa partie inférieure.

Calgul de l'Amplitude de la hovle produtte :

Le volet étant plan, si sa position moyenne est verticale, la théorie exposée dans cet article permet de calculer l'amplitude de la houle produite; il suffit de superposer les solutions obtenues pour le batteur à simple articulation et le batteur piston.
On obtient ainsi pour la demi-amplitude :

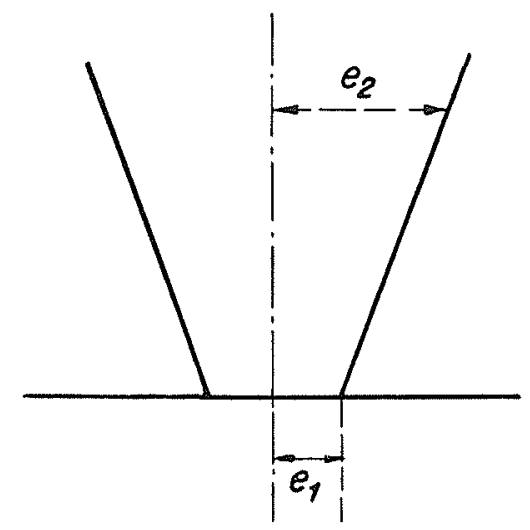

$$
\begin{gathered}
a=2\left(e_{2}-e_{1}\right) \frac{(1-\operatorname{ch} m h+m h \operatorname{sh} m h) \operatorname{sh} m h}{(\operatorname{sh} m h \operatorname{ch} m h+m h) m h} \\
+2 e_{1} \frac{\operatorname{sh}^{2} m h}{\operatorname{sh} m h \operatorname{ch} m h+m h}
\end{gathered}
$$

INERTIE :

Elle n'est sans doute pas plus grande que celle du volet à simple articulation.

\section{RÉGLAGE :}

La course des extrémités supérieure et inférieure du volet se règle en déplaçant le point d'articulation des bielles sur les bras oscillants.

L'amplitude de la houle se règle en agissant sur l'excentricité du maneton ou la course du piston. 
mensions limitées, il peut être intéressant de chercher à réaliser une houle qui soit aussi parfaite que possible dès le départ du batteur. L'emploi d'un appareil à vagues relativement complexe devient alors parfaitement justifié.

$2^{\circ}$ Même si nous supposons que l'on puisse disposer sans inconvénient de la longueur nécessaire pour que la perturbation initiale s'élimine, il n'en est pas moins vrai que la théorie présentée n'est approchée qu'au premier ordre. C'est à dire que la théorie nous montre seulement que tous les batteurs à mouvement sinusoïdal sont à peu près équivalents pour les houles de faible cambrure (et l'on constate d'ailleurs expérimentalement qu'il est effectivement très facile de faire de belles houles de faible cambrure), par contre cette théorie ne nous permet plus de conclure en ce qui concerne les houles d'amplitude notable (1).

Or, l'expérience nous apprend effectivement que les houles de cambrure notable crées par les batteurs sont loin d'ètre toutes de la mème qualité, même si on les observe après un parcours important. La présente théorie a donc pour intérêt de montrer que ces défauts ne sont pas nécessairement dus au fait que le batteur utilisé diffère notablement du batteur idéal.

Cependant le caractère plus négatif que constructif de cette conclusion fait que des études expérimentales restent encore nécessaires. Les résultats théoriques déjà connus seront d'ailleurs très utiles pour l'interprétation des observations et l'orientation des recherches ultérieures.

Par exemple, l'expérience nous montre que pour un batteur et une cambrure donnés les houles sont d'autant plus belles qu'elles sont plus courtes. Ce résultat ne peut être expliqué rigoureusement par notre calcul, mais il peut cependant recevoir une interprétation admissible. Nous avons vu en effet qu'un batteur piston, de même d'ailleurs qu'un volet battant articulé sur le fond, devait avoir, au voisinage de la surface, une anplitude moitié moindre que celle de la houle, si celle-ci était courte, et au contraire une amplitude plusieurs fois supérieure à celle de la houle, si celle-ci était longue. On concoit donc que la condition de petitesse des mouvements soit mieux réalisée dans le premier cas que dans le second, ce qui pourrait donner une explication plausible de la différence de qualité des houles observées. Si cette façon de voir était juste, il semblerait logique d'en déduire que le meilleur batteur est toujours le batteur «piston $»$. Mais il ne faut pas

(1) Cette insuffisance de la theorie n'est d'ailleurs pas un argument en faveur du batteur idéal puisque ce demier n'est également justifié que par une théorie du premier ordre, ainsi que nous l'avons déjà souligné plus haut. oublier que ce type de batteur donne lieu à des surpressions d'inertie assez importantes et que, par conséquent, on ne peut en recommander l'emploi sans réserves.

Enfin la théorie nous offre un guide appréciable dans le choix des moyens permettant de remédier aux défauts des batteurs des types étudiés, lorsque ces défauts subsistent, même quand la réalisation du batteur est parfaitement conforme aux schémas que nous étudions ici. Elle nous montre en effet que, si l'on se limite aux théories du premier ordre, il n'y a pas de raison théorique de rechercher la perfection des houles dans une imitation aussi précise que possible du batteur idéal et que, par conséquent, les défauts qui subsistent proviennent de phénomènes d'ordre supérieur au premier. Ainsi elle oriente notre attention vers d'autres solutions. Parmi celles-ci, la plus naturelle consiste à modifier ${ }^{\circ}$ la loi de mouvement du batteur. Cette idée paraît d'ailleurs intéressante car l'expérience semble montrer que les déformations qui persistent même loin du batteur sont vraisemblablement dues à l'émission d'harmoniques de la houle fondamentale. Ces harmoniçues sont des houles de même nature aue la houle fondamentale de période $\mathrm{T}$, mais elles ont des périodes $\mathrm{T} / 2, \mathrm{~T} / 3$, $\mathrm{T} / 4$, etc. On conçoit que cette émission parasite puisse être compensée par une modification complémentaire du mouvement du batteur.

Il semble donc logique d'escompter que les derniers défauts des batteurs puissent être supprimés par le choix d'une loi de mouvement différant plus ou moins de la loi sinusoïdale. Nous n'insistons pas sur ce point qui nous entrainerait trop loin; signalons simplement que des études expérimentales sont en cours à ce sujet au Laboratoire Neyrpic.

$3^{\circ}$ La présente théorie ne tient évidemment pas compte des défauts de réalisation éventuels du batteur. Ceux-ci sont pourtant loin d'être toujours négligeables, signalons les principaux :

\section{a) Limperfection du mouvement du batteur.}

En plus de tous les défauts de conception ou d'exécution des organes mécaniques, il faut souligner l'extrême importance de l'inertie des masses oscillantes. A cause de cette inertie, l'effort que doit fournir le moteur risque d'être soumis à des fluctuations considérables et il devient difficile d'assurer la régularité de son fonctionnement.

\section{b) Le défaut d'étanchéité.}

Nous avons déjà dit un mot de cette auestion plus haut, précisons cependant que si lés différences de pression, de part et d'autre du batteur, (Voir suite page 496.) 


\section{FICHE No 10}

\section{Volet du "Beach Erosion Board"}

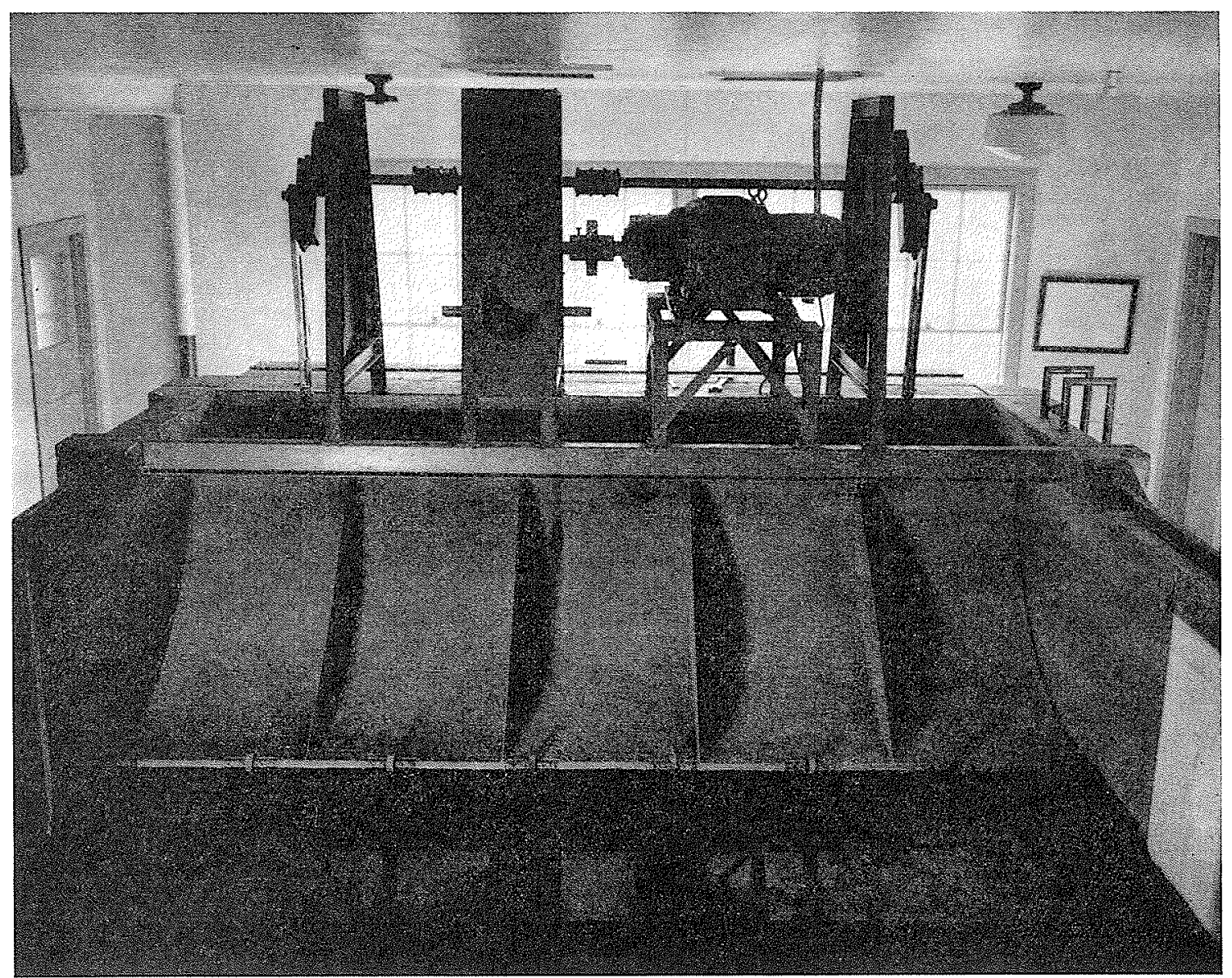

Principe. - Lor du movvement.

G'est un volet courbe à double articulation portant au fond du canal sur deux glissières et un certain nombre de galets; il est mis en mouvement par deux bielles qui l'attaquent par la face arrière.
Calcul de l'amplitude :

L'amplitude ne peut etre calculée d'après la théorie exposée dans cet article car la forme du batteur, dans sa position moyenne, n'est pas un plan vertical. 


\section{FICHE No 11}
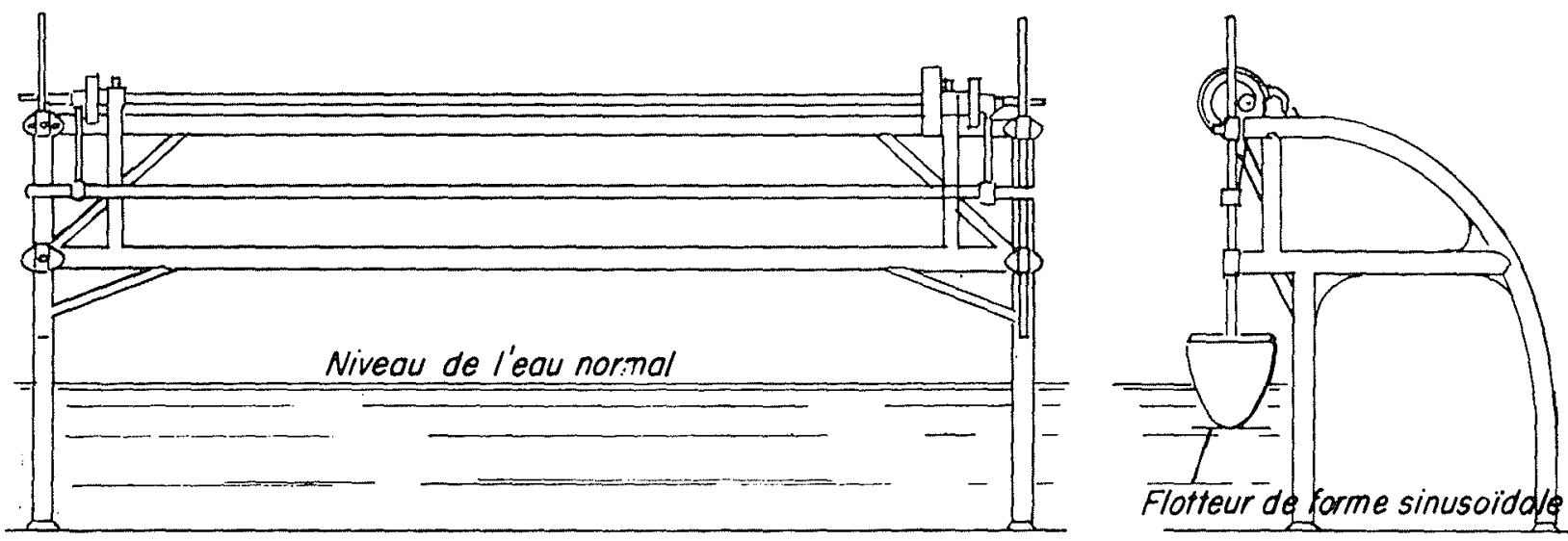

Extrait de : L. Bonnex et Y. LAmoen: «Etude des ports belges de la mer du Nord. Essais sur modèle réduit ». Annales des Travaix publics de Belgique, juin, août, octobre et décembre 1948

\section{Plongeurs}

\section{Principe. - Lor de Mouvement.}

Un corps de forme donnée est animé d'un mouvement alternatif vertical d'immersion et d'émersion.

I.a forme du plongeur peut être étudiée de facon a obtenir des trajectoires de particules très voisines de celles de la houle; cependant un prisme triangulaire donne déjà de bons résultats.

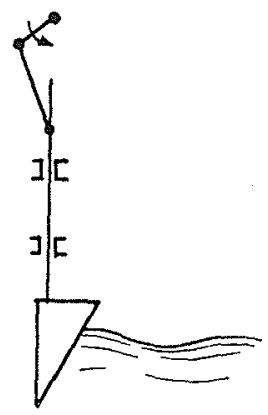

CALCUL DE L'AMPLITUDE :

Pour un plongeur de forme quelconque, l'amplitude ne peut être calculée au moyen de la théorie donnée dans cet article. Toutefois, pour le prisme triansulaire, si l'angle de la face oblique avec la verticale est faible, on peut assimiler les positions successives de cette dernière a celles d'un volet plan.

\section{Construction :}

I a construction est relativement simple; elle l'est toutefois moins que celle du volet à simple articulation. Cependant, dans certains cas, ce type de batteur exige la présence d'une fosse, ce qui complique la construction du modèle sur lequel il est employé.

\section{ENTRETIEN :}

Le batteur est tres robuste; de plus, comme il n'existe aucune articulation dans l'eau, l'entretien est très faible.

\section{INERTIE :}

L'inertie est assez grande; l'appareil peut exiger la présence d'un volant.

\section{POUVORR RÉFLÉCHISSANT:}

Le batteur est réfléchissant et peut nécessiter l'emploi d'un « filtre ».

\section{Réglage :}

Le réglage de l'amplitude se fait très commodément et dans un domaine assez large en modifiant la course du plongeur.

Le réglage de la période se fait en modifiant la vitesse du moteur.

\section{MoBilité :}

Du fait de sa simplicité, ce type de batteur peut ctre monté de facon à ètre orienté assez facilement.

La figure montre un ensemble monté sur galets qui peut être disposé en tout point d'un modèle et dans la direction désirée, à condition que les houles n'aient pas une amplitude et une longueur d'onde trop considérables. 


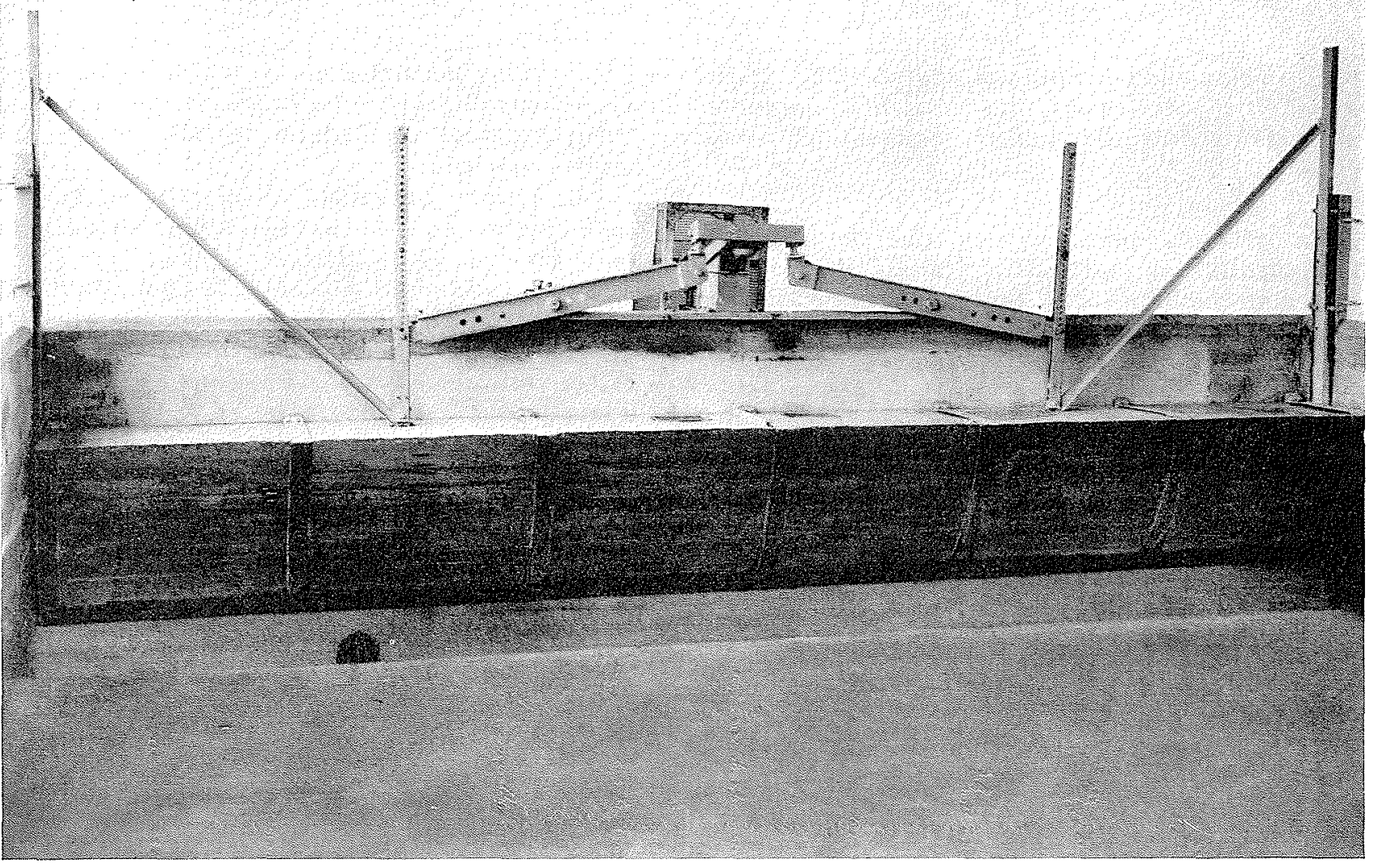

\section{FICHE No 11 (SUITE)}

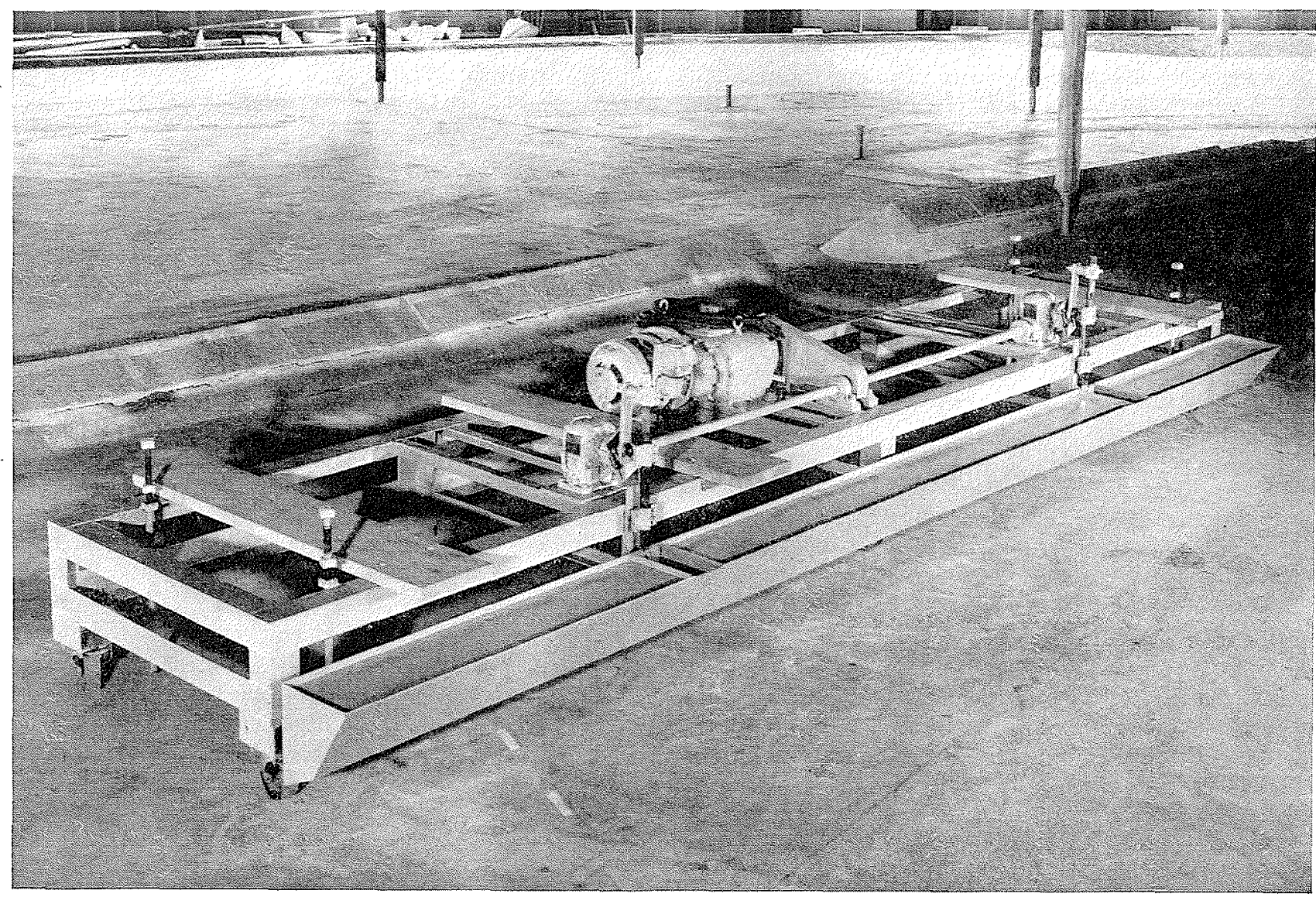

Photographies communiquées par le U.S. Waterways Experiment Station, Vicksburg, U.S.A. 
varient sinusoïdalement, par contre la variation du débit des fuites est plus complexe et risque ainsi d'introduire des houles harmoniques parasites.

En résumé, nous voyons que le résultat fondamental de notre étude a été de donner au constructeur du batteur une latitude extrêmement vaste dans le choix des types d'appareils. Nous l'avons affranchi en particulier du souci de se conformer le plus possible au modèle du batteur idéal. Par contre, nous lui avons créé le souci de calculer un batteur nouveau. En effet, il ne faut pas oublier qu'un des avantages essentiels du batteur parfait était que l'on pouvait calculer, grâce aux équations de LAPLACE, à la fois l'amplitude qu'il fallait lui donner pour obtenir telle ou telle houle, et la répartition des pressions qu'il aurait à vaincre.

La présente théorie annule ce dernier avantage puisqu'elle nous permet de calculer ces éléments dans le cas d'un batteur de forme quelconque.
Elle nous permet donc de calculer les pièces du batteur de façon à leur assurer une résistance suffisante tout en évitant de leur donner une inertie superflue.

Elle permet également de déterminer l'amplitude que le mouvement du batteur devra avoir pour créer la houle désirée.

Elle permet enfin d'orienter le choix d'un type de batteur en fonction de deux données nouvelles qui sont l'amplitude des perturbations initiales et l'amplitude des fluctuations de pression d'inertie.

La théorie hydrodynamique au premier ordre des batteurs permet done d'accomplir un premier pas vers la connaissance des conditions de fonctionnement des appareils à houle employés en laboratoire. Des résultats plus complets pourraient être obtenus par une théorie approchée au second ordre ou par des essais systématiques; nous ne pouvons que souhaiter que de tels travaux soient entrepris dans un proche avenir.

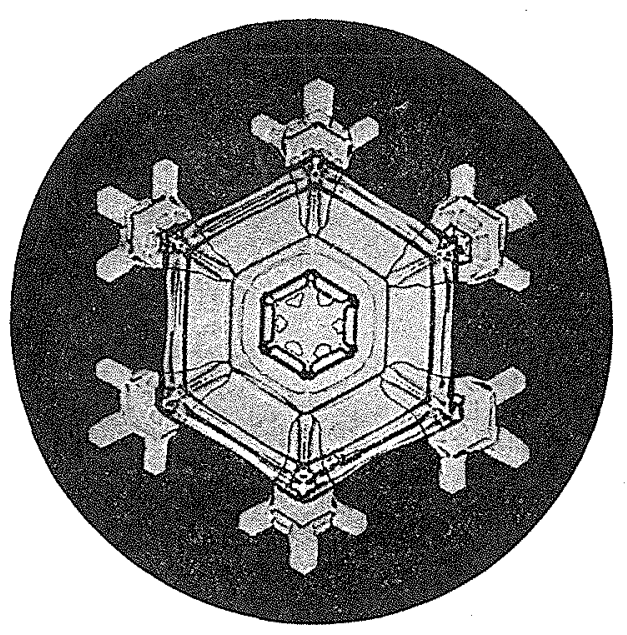

(A suivie.) 